\title{
First CMB Constraints on the Inflationary Reheating Temperature
}

\author{
Jérôme Martin* \\ Institut d'Astrophysique de Paris, \\ UMR 7095-CNRS, Université Pierre et Marie Curie, \\ 98bis boulevard Arago, 75014 Paris, France \\ Christophe Ringevali \\ Institute of Mathematics and Physics, Centre for Particle Physics and Phenomenology, \\ Louvain University, 2 Chemin du Cyclotron, 1348 Louvain-la-Neuve (Belgium)
}

(Dated: October 8, 2018)

\begin{abstract}
We present the first Bayesian constraints on the single field inflationary reheating era obtained from Cosmic Microwave Background (CMB) data. After demonstrating that this epoch can be fully characterized by the so-called reheating parameter, we show that it is constrained by the seven years Wilkinson Microwave Anisotropies Probe (WMAP7) data for all large and small field models. An interesting feature of our approach is that it yields lower bounds on the reheating temperature which can be combined with the upper bounds associated with gravitinos production. For large field models, we find the energy scale of reheating to be higher than those probed at the Large Hadron Collider, $\rho_{\text {reh }}^{1 / 4}>17.3 \mathrm{TeV}$ at $95 \%$ of confidence. For small field models, we obtain the twosigma lower limits $\rho_{\text {reh }}^{1 / 4}>890 \mathrm{TeV}$ for a mean equation of state during reheating $\bar{w}_{\text {reh }}=-0.3$ and $\rho_{\text {reh }}^{1 / 4}>390 \mathrm{GeV}$ for $\bar{w}_{\text {reh }}=-0.2$. The physical origin of these constraints is pedagogically explained by means of the slow-roll approximation. Finally, when marginalizing over all possible reheating history, the WMAP7 data push massive inflation under pressure $(p<2.2$ at $95 \%$ of confidence where $p$ is the power index of the large field potentials) while they slightly favor super-Planckian field expectation values in the small field models.
\end{abstract}

PACS numbers: $98.80 . \mathrm{Cq}, 98.70 . \mathrm{Vc}$

\section{INTRODUCTION}

The current ongoing flow of high accuracy astrophysical observations has important consequences for our understanding of the very early Universe. In particular, the widely accepted inflationary paradigm [1-4] (for a review, see e.g. Refs. [5 8]) is now under close scrutiny. According to this scenario, the Cosmic Microwave Background (CMB) anisotropies and the large scale structures originate from the unavoidable quantum fluctuations of the inflaton and gravitational fields in the very early Universe subsequently amplified during inflation [9-13]. One can show that the corresponding power spectrum of the cosmological fluctuations naturally acquires an almost scale invariant form which is fully consistent with all observations. Another crucial property of the inflationary power spectrum is that the slight deviations from scale invariance are linked to the microphysics of inflation [14-16]. Therefore, by measuring these deviations, one can probe the shape of the inflaton potential and, therefore, learn about the physical origin of the inflaton field.

It is often claimed from the above properties that observations give access to a limited part of the potential only, namely the one which is slow-rolled over by the inflaton when scales of astrophysical interest today left

*Electronic address: jmartin@iap.fr

${ }^{\dagger}$ Electronic address: christophe.ringeval@uclouvain.be the Hubble radius. This observational window represents a range of approximately 7 e-folds or three decades in wavenumbers. However, inflation does not consist of the slow-roll phase only and the pre and/or reheating period is also of fundamental importance since it allows us to understand how inflation is connected to the hot big-bang phase [17 20]. This physical phenomenon is related to a different part of the inflationary potential, usually the one located close to its true minimum, i.e. a few decades in e-folds away from the observable window.

Observation of pre-/reheating effects can be achieved in two ways. First, the power spectrum can evolve on large scales when the inflaton field oscillates around the minimum of its potential. However, this happens only in quite complicated models, typically those containing more than one field 21 23]. In fact, it was recently shown that this type of effect can also happen in single field inflation but on much smaller scales 2426$]$. Second, the duration of the pre-/reheating phase can significantly modify the position of the observational window mentioned above. Put differently, at fixed astrophysical scales today, changing the pre-/reheating duration is equivalent to moving the window along the potential, hence probing different values of the power spectrum spectral index, amplitude of the anisotropies and tensor-to-scalar ratio. Obviously, this cannot be done arbitrarily because CMB data impose accurate bounds on their value. Conversely, this opens up the possibility to constrain the pre-/reheating duration and/or its equation of state from CMB data [27]. Notice that a direct 
detection of primordial gravitational waves would also allow us to probe the reheating temperature, as shown in Refs. [28, 29].

The goal of this article is to address this question for the standard scenarios of inflation. It is traditional to study three categories of models usually considered as representative of the full inflationary space. These models are large field [30], small field [2, 3], and hybrid inflation [31]. Hybrid scenarios involve multiple fields and, therefore, the power spectrum can change during the preheating phase. This makes this class of scenarios deserving of a separate investigation. For this reason, in this article, we limit ourselves to the class of large and small field models.

In the following, we will use the term "reheating" to refer to the pre-/reheating phases of the Universe defined to have occurred just after the end of inflation and just before the radiation dominated era. So far, the constraints on the reheating energy scale are not so numerous. Obviously, it should be less than the energy scale of inflation which implies that $T_{\text {reh }} \lesssim 10^{16} \mathrm{GeV}$. In addition, if one assumes that supersymmetry is the correct extension of the standard model of particle physics, then constraints from Big-Bang Nucleosynthesis (BBN) on unstable gravitinos lead to a reheating temperature $T_{\text {reh }} \lesssim 10^{7} \mathrm{GeV}[32$ 42]. Notice that this constraint can nevertheless be avoided if one considers the scenario of Ref. [43]. Reheating itself should also proceed before BBN and this implies that $T_{\text {reh }} \gtrsim 10 \mathrm{MeV}$. We see that the reheating temperature is poorly constrained, in particular, its lower limit. As a matter of fact, the work presented here precisely yields a lower limit on the reheating energy scale from the current seven years Wilkinson Microwave Anisotropies Probe (WMAP7) data [44 46].

In order to derive constraints on the reheating phase, we make use of Bayesian techniques and utilize a full numerical approach [47]. This has several advantages. First, it is exact and rests only on the linear theory of cosmological perturbations: the method remains accurate when the slow-roll approximation breaks down, as one expects near the end of inflation. Second, and of particular importance for the present work, it permits a new treatment of reheating. Indeed, instead of viewing the reheating parameters as nuisance parameters, they can easily be included in the Bayesian data analysis process. Third, the evolution of cosmological perturbations in the hot big-bang eras already relies on numerical codes. Treating perturbations during inflation in the same way allows the whole procedure to be automatized and to be easily extended to other scenarios. Fourth, the numerical approach allows us to address the question of the priors choice in a particularly well-defined way. Indeed, from a physical point of view, our prior knowledge is on the inflationary theory and not on the shape of the primordial power spectra which is actually a model prediction. Therefore, it is better, and easier, to choose prior probability distributions directly on the model parameters, such as the power index of the large field potentials.
This reflects the fact that a model of inflation is not a disembodied mathematical structure that one only needs to "fit" but a physical scenario rooted in high energy physics that one needs to understand.

This paper is organized as follows. In Sec. III we extend the above discussion and explain in detail why the reheating epoch can be constrained with CMB data. In particular, we introduce the so-called reheating parameter which depends on the reheating duration and on the mean equation of state of the fluid dominating the Universe during this epoch. Then, using the slow-roll approximation, we analytically demonstrate that the accuracy of the WMAP7 data is now sufficient to obtain some constraints on the reheating era. In Sec. III, using a full numerical integration of the tensor and scalar power spectra coupled to Bayesian methods, we derive the constraints that any reheating model has to satisfy. Then, assuming specific values for the mean equation of state, we translate these constraints into new lower limits for the reheating energy density and/or reheating temperature. These results significantly improve the bounds coming from the Big-Bang Nucleosynthesis. In Sec. IV] we recap our main findings and discuss how our results are modified by the inclusion of others CMB data sets. In Appendix A. we work out a typical example which illustrates the robustness of our assumptions: a noninstantaneous transition between reheating and the radiation dominated era when one considers the finite decay width of the inflaton field. Finally, as a by-product of our data analysis, Appendix B presents the updated WMAP7 constraints on the spectral index, tensor-to-scalar ratio and first order slow-roll parameters marginalized over second order effects.

\section{PHYSICAL ORIGIN OF THE CONSTRAINT}

Before presenting and discussing the constraints on the reheating temperature, we explain why and how these ones can be inferred from high accuracy CMB observations. In particular, we use the slow-roll approximation to explicitly illustrate the method.

\section{A. Parametrizing the reheating}

The evolution of scalar (density) perturbations is controlled by the so-called Mukhanov-Sasaki variable $v_{\boldsymbol{k}}$. If matter is described by a scalar field (as is the case during inflation and pre-/reheating), then its equation of motion is given, in Fourier space, by [6 6 , 15$]$

$$
v_{\boldsymbol{k}}^{\prime \prime}+\left[k^{2}-\frac{\left(a \sqrt{\epsilon_{1}}\right)^{\prime \prime}}{a \sqrt{\epsilon_{1}}}\right] v_{\boldsymbol{k}}=0 .
$$

Here, a prime denotes a derivative with respect to conformal time. The quantity $k$ is the comoving wave number and $\epsilon_{1} \equiv-\dot{H} / H^{2}$ is the first Hubble flow func- 
tion [48], $H=\dot{a} / a$ being the Hubble parameter and $a$ the Friedmann-Lemaître-Robertson-Walker (FLRW) scale factor (a dot means derivative with respect to cosmic time). The quantity $v_{\boldsymbol{k}}$ is related to the curvature perturbation $\zeta_{\boldsymbol{k}}$ through the following expression:

$$
\zeta_{\boldsymbol{k}}=\frac{1}{M_{\mathrm{P} l}} \frac{v_{\boldsymbol{k}}}{a \sqrt{2 \epsilon_{1}}}
$$

where $M_{\mathrm{Pl}}$ stands for the reduced Planck mass. As a consequence, the power spectrum of $\zeta_{\boldsymbol{k}}$ can be expressed as

$$
\mathcal{P}_{\zeta}(k) \equiv \frac{k^{3}}{2 \pi^{2}}\left|\zeta_{\boldsymbol{k}}\right|^{2}=\frac{k^{3}}{4 \pi^{2} M_{\mathrm{P} l}^{2}}\left|\frac{v_{\boldsymbol{k}}}{a \sqrt{\epsilon_{1}}}\right|^{2} .
$$

In order to calculate $\mathcal{P}_{\zeta}(k)$, one needs to integrate Eq. (1), which requires the knowledge of the initial conditions for the mode function $v_{\boldsymbol{k}}$. Since, at the beginning of inflation, all the modes of astrophysical interest today were much smaller than the Hubble radius, the initial conditions are chosen to be the Bunch-Davis vacuum which amounts to

$$
\lim _{k / \mathcal{H} \rightarrow+\infty} v_{k}=\frac{1}{\sqrt{2 k}} \mathrm{e}^{-i k \eta},
$$

where $\eta$ denotes conformal time and $\mathcal{H}=a H$ is the conformal Hubble parameter. The importance of the curvature perturbation lies in the fact that it is directly related to CMB anisotropies, the two point correlation function of which can be expressed in term of the spectrum of $\zeta_{\boldsymbol{k}}$. Moreover, under very general conditions (including the assumption that inflation proceeds with only one field), $\zeta_{\boldsymbol{k}}$ is a conserved quantity on large scales and, therefore, can be used to propagate the inflationary spectrum from the end of inflation to the post-inflationary era [49]. In other words, the power spectrum is not affected by the post-inflationary evolution, in particular by the pre/reheating epoch.

However, this does not mean that the reheating era has no effect on the inflationary predictions. On the contrary, the relation between the physical scales at present time and during inflation depends on the properties of this phase of evolution. As a consequence, in order to calibrate the inflationary spectrum with respect to the physical scales of astrophysical interest today, it is necessary to know how the reheating phase proceeded. Conversely, this also opens the possibility to constrain the physical conditions that prevailed at that time by means of CMB observations.

In order to put the above considerations on a quantitative footing, let us rewrite Eq. (11) in terms of the number of e-folds during inflation, $N \equiv \ln \left(a / a_{\text {ini }}\right)$, where $a_{\text {ini }}$ is the value of the scale factor at the beginning of inflation. It takes the form

$$
\frac{\mathrm{d}^{2} v_{\boldsymbol{k}}}{\mathrm{d} N^{2}}+\frac{1}{\mathcal{H}} \frac{\mathrm{d} \mathcal{H}}{\mathrm{d} N} \frac{\mathrm{d} v_{\boldsymbol{k}}}{\mathrm{d} N}+\left[\left(\frac{k}{\mathcal{H}}\right)^{2}-U_{\mathrm{S}}(N)\right] v_{\boldsymbol{k}}=0,
$$

where $U_{\mathrm{S}}(N)$ is an effective potential for the perturbations which depends on the scale factor and its derivatives only. All the terms in this equation but $k / \mathcal{H}$ are completely specified by the inflationary background evolution. In practice, we are given a physical scale today, say $k / a_{\text {now }}$ (for instance $k / a_{\text {now }}=0.05 \mathrm{Mpc}^{-1}$ ) and we need to express $k / \mathcal{H}$ in terms of $k / a_{\text {now }}$ and quantities defined during inflation. Straightforward considerations lead to

$$
\frac{k}{\mathcal{H}}=\frac{\Upsilon_{\boldsymbol{k}}}{H(N)} \mathrm{e}^{N_{\mathrm{T}}-N},
$$

where $N_{\mathrm{T}}$ is the total number of e-folds during inflation and $\Upsilon_{\boldsymbol{k}}$ is defined by

$$
\Upsilon_{k} \equiv \frac{k}{a_{\text {now }}}\left(1+z_{\text {end }}\right),
$$

with $z_{\text {end }}$ being the redshift of the end of inflation. As expected $\Upsilon_{\boldsymbol{k}}$ depends on the whole post-inflationary history through $z_{\text {end }}$. During this post-inflationary history, only the reheating phase is poorly known and represents, by far, the main source of uncertainty for the inflationary predictions. For convenience, we rewrite $\Upsilon_{\boldsymbol{k}}$ as

$$
\Upsilon_{k}=\frac{k}{a_{\text {now }}}\left(\frac{\rho_{\text {end }}}{\Omega_{\gamma} \rho_{\text {cri }}}\right)^{1 / 4} R_{\text {rad }}^{-1},
$$

thus defining the new parameter $R_{\text {rad }}$. This parameters plays a crucial role in this article. In the above equation, $\rho_{\text {end }}$ is the energy density at the end of inflation, $\rho_{\text {cri }}$ is the present day critical energy density and $\Omega_{\gamma} \simeq 2.471 \times 10^{-5} h^{-2}$ is the density parameter of radiation today. As a result $\Omega_{\gamma} \rho_{\text {cri }} \equiv \rho_{\gamma}$ is the present day radiation energy density and does not depend on $h^{2}$. The above equations make clear that the parameter $R_{\text {rad }}$ must be specified if one wants to compare an inflationary model to observations.

In fact, the quantity $R_{\text {rad }}$ has a simple physical interpretation. Let us assume that the reheating phase is dominated by a conserved effective fluid with energy density $\rho$ and pressure $P$. The fact that we assume the effective fluid to be conserved is not a limitation. For instance, in a simple model where the inflaton scalar field is coupled to radiation (see Appendix $\bar{A}$ ), the effective fluid is just defined by $\rho=\rho_{\phi}+\rho_{\gamma}$ and $P=P_{\phi}+\rho_{\gamma} / 3$. The scalar field and the radiation are not separately conserved but the effective fluid is. Then, it is straightforward to show that

$$
\rho(N)=\rho_{\text {end }} \exp \left\{-3 \int_{N_{\mathrm{T}}}^{N}\left[1+w_{\mathrm{reh}}(n)\right] \mathrm{d} n\right\},
$$

where $w_{\text {reh }} \equiv P / \rho$ is the equation of state function during reheating. Using this expression, one obtains

$$
\ln R_{\mathrm{rad}}=\frac{\Delta N}{4}\left(-1+3 \bar{w}_{\mathrm{reh}}\right)
$$


where

$$
\Delta N \equiv N_{\text {reh }}-N_{\mathrm{T}}
$$

is the total number of e-folds during reheating, $N_{\text {reh }}$ being the number of e-folds at which reheating is completed and the radiation dominated era begins. The quantity $\bar{w}_{\text {reh }}$ stands for the mean equation of state parameter

$$
\bar{w}_{\text {reh }} \equiv \frac{1}{\Delta N} \int_{N_{\mathrm{T}}}^{N_{\mathrm{reh}}} w_{\mathrm{reh}}(n) \mathrm{d} n
$$

Therefore, the parameter $R_{\text {rad }}$ only depends on what happens during reheating. To put differently, it singles out in the expression of $\Upsilon_{k}$, the contribution coming from reheating. Equation (10) also allows us to understand why $R_{\text {rad }}$ carries the subscript "rad". Indeed, if the effective fluid is equivalent to radiation, then $\bar{w}_{\text {reh }}=1 / 3$ and $\ln R_{\text {rad }}=0$. The physical interpretation is very clear: in this case the reheating stage cannot be distinguished from the subsequent radiation dominated era and, therefore, cannot affect the inflationary predictions: as a consequence $R_{\mathrm{rad}}=1$ in Eq. (8).

In fact, one can even go further and express $R_{\text {rad }}$ in an even more compact form. Using Eq. (9), one can write $\rho_{\text {reh }}=\rho_{\text {end }} \exp \left[-3 \Delta N\left(1+\bar{w}_{\text {reh }}\right)\right]$ from which, together with Eq. (10), one obtains

$$
\ln R_{\mathrm{rad}}=\frac{1-3 \bar{w}_{\mathrm{reh}}}{12\left(1+\bar{w}_{\mathrm{reh}}\right)} \ln \left(\frac{\rho_{\mathrm{reh}}}{\rho_{\mathrm{end}}}\right)
$$

where $\rho_{\text {reh }}$ has to be understood as the energy density at the end of the reheating era, i.e. $\rho\left(N_{\text {reh }}\right)$.

Let us summarize our discussion. In order to calculate the power spectrum of the inflationary cosmological perturbations, one needs to solve Eq. (5). In this formula, all the terms are accurately known during inflation except

$$
\frac{k}{\mathcal{H}}=\frac{k}{a_{\text {now }}}\left(\frac{\rho_{\text {end }}}{\rho_{\gamma}}\right)^{1 / 4} \frac{1}{H(N) R_{\text {rad }}} \mathrm{e}^{N_{\mathrm{T}}-N},
$$

and the theoretical uncertainty in this expression solely comes from the parameter $R_{\text {rad }}$ which depends on reheating only (more precisely, on the energy density at the end of reheating, $\rho_{\text {reh }}$, and the mean equation of state $\bar{w}_{\text {reh }}$ ).

\section{B. Why CMB observations constrain reheating}

Having discussed the physical interpretation of $R_{\text {rad }}$, we now explain how the CMB observations can constrain its value. For this purpose, we reexpress $R_{\text {rad }}$ in terms of quantities defined at the Hubble radius crossing. One obtains

$$
\begin{aligned}
\ln R_{\mathrm{rad}} & =N_{\mathrm{T}}-N_{*}+N_{0}-\frac{1}{4} \ln \left(\frac{H_{*}^{2}}{M_{\mathrm{P} l}^{2} \epsilon_{1 *}}\right) \\
& +\frac{1}{4} \ln \left(\frac{3}{\epsilon_{1 *}} \frac{V_{\mathrm{end}}}{V_{*}} \frac{3-\epsilon_{1 *}}{3-\epsilon_{1 \mathrm{end}}}\right)
\end{aligned}
$$

where we have defined

$$
N_{0} \equiv \ln \left(\frac{k / a_{\text {now }}}{\rho_{\gamma}^{1 / 4}}\right)
$$

In this formula, $N_{*}$ is the e-folds number at which the scale $k / a_{\text {now }}$ crossed out the Hubble radius during inflation (all the quantities with a subscript "*" are evaluated at that time) and $V(\phi)$ is the inflaton potential. Despite the appearance of the first Hubble flow function, this equation is exact (moreover, we also have $\epsilon_{1 \mathrm{end}}=1$ ). At leading order, one has

$$
\frac{H_{*}^{2}}{M_{\mathrm{P} l}^{2} \epsilon_{1 *}}=8 \pi^{2} P_{*}
$$

where the amplitude of the scalar power spectrum at the pivot scale $P_{*}=\mathcal{P}_{\zeta}\left(k_{*}\right)$ is directly related to the Cosmic Background Observer (COBE) normalization.

The above equation (15) can be used in two different manners. The first way is to assume something about $R_{\text {rad }}$ and to derive the corresponding range of variations of the inflationary slow-roll predictions $N_{*}$ and $\epsilon_{i}\left(N_{*}\right)$. In other words, this determines how the inflationary predictions depend on the details of the reheating era. This approach is the one usually considered in the literature to compare inflationary predictions to the current constraints on the slow-roll parameters $\epsilon_{i *}$ (or spectral index and tensor-to-scalar ratio). Unfortunately, the assumptions on $R_{\text {rad }}$ are rarely explicit and comparison is only made by choosing reasonably assumed values of $N_{*}$ : typically 30 and 60 e-folds as one may derive under generic assumptions [50]. However, as Eq. (15) explicitly shows, once $V(\phi)$ is chosen, and the tilt and amplitude of the scalar perturbations measured, $N_{*}$ is directly related to $R_{\text {rad }}$, which itself, as already noticed, depends on the energy density $\rho_{\text {reh }}$ at which reheating ends and $\bar{w}_{\text {reh }}$. As a result, the range of variation for $N_{*}$ can only be known once a reheating model is assumed. Without such an assumption, from one model to another, an assumed value of $N_{*}$ may inconsistently imply that the reheating occurs after nucleosynthesis, or even at energy densities higher than $\rho_{\text {end }}$. This type of model would therefore appears to be compatible with the CMB data favored power spectra while being totally inconsistent with standard cosmology.

Let us now see how it works in practice. In order to be consistent with the standard cosmological model, $\ln R_{\mathrm{rad}}$ cannot take arbitrary values. One should have $\bar{w}_{\text {reh }}<$ 1 to respect the positivity energy conditions of General Relativity and $\bar{w}_{\text {reh }}>-1 / 3$ by the very definition of reheating which is not inflation. Notice that we impose conditions on the mean value of the equation of state only. In addition, reheating should occur after inflation and before $\mathrm{BBN}$, i.e. $\rho_{\text {nuc }}<\rho_{\text {reh }}<\rho_{\text {end }}$, with

$$
\rho_{\text {nuc }} \equiv(10 \mathrm{MeV})^{4} \text {. }
$$

This allows us to explicitly use Eq. (15). Combined with Eq.(13), we can determine the range of variation 
of $\Delta N_{*} \equiv N_{\mathrm{T}}-N_{*} \in\left[\Delta N_{*}^{\text {nuc }}, \Delta N_{*}^{\text {end }}\right]$. Straightforward manipulations lead to

$$
\begin{aligned}
\Delta N_{*}^{\mathrm{nuc}}= & -N_{0}+\ln \left(\frac{H_{*}}{M_{\mathrm{P} l}}\right)-\frac{1}{3\left(1+\bar{w}_{\mathrm{reh}}\right)} \ln \frac{\rho_{\mathrm{end}}}{M_{\mathrm{P} l}^{4}} \\
& +\frac{1-3 \bar{w}_{\mathrm{reh}}}{12\left(1+\bar{w}_{\mathrm{reh}}\right)} \ln \frac{\rho_{\mathrm{nuc}}}{M_{\mathrm{P} l}^{4}},
\end{aligned}
$$

while if one chooses $\rho_{\text {reh }}=\rho_{\text {end }}$, one obtains

$$
\Delta N_{*}^{\mathrm{end}}=-N_{0}+\ln \left(\frac{H_{*}}{M_{\mathrm{P} l}}\right)-\frac{1}{4} \ln \frac{\rho_{\mathrm{end}}}{M_{\mathrm{P} l}^{4}} .
$$

Interestingly enough, the last equation no longer depends on $\bar{w}_{\text {reh }}$. This is of course because requiring $\rho_{\text {reh }}=\rho_{\text {end }}$ means that one immediately reheats the Universe after inflation. It is important to notice that these equations are algebraic for $\Delta N_{*}^{\text {nuc }}$ and $\Delta N_{*}^{\text {end }}$ because $H_{*}$ and $\rho_{\text {end }}$ are also functions of $\Delta N_{*}$. The corresponding range of variations of the inflationary predictions is determined by calculating $\Delta \epsilon_{i *}=\epsilon_{i}\left(\Delta N_{*}\right)$ with $\Delta N_{*}$ given above. To proceed further, one needs to specify the model of inflation. In the next section, one considers the prototypical scenario of chaotic inflation as well as the small field models.

Before dealing with these explicit examples, let us briefly anticipate and discuss the second way of using Eq. (15). It consists of considering $R_{\text {rad }}$ as an observable model parameter and in including it in the data analysis, as it should be from a Bayesian point of view. If we are given a specific potential, then $V_{\text {end }}$ is explicitly known. CMB data put a limit on $H_{*}^{2} / \epsilon_{1 *}$ through the amplitude of the anisotropies, as well as on $\epsilon_{1 *}$ from the tensor-toscalar ratio. As a result, one expects CMB data to also give some information on $R_{\mathrm{rad}}$. This is the subject of Sec. III in which we perform a Bayesian data analysis of the WMAP7 data for both the large and small field models by including the reheating. For the first time, we find that $R_{\text {rad }}$ is not only a nuisance parameter for inflation but ends up being constrained by the WMAP7 data. We then discuss the physical implications of these bounds and show that CMB data give us a lower bound on the energy scale at which the reheating ended.

\section{Large field models}

We now consider the archetypal model of inflation, namely, large field inflation. This working example is important because it allows us to show explicitly which type of constraints one should expect. Large field models are characterized by the potential

$$
V(\phi)=M^{4}\left(\frac{\phi}{M_{\mathrm{Pl}}}\right)^{p},
$$

where $M$ is an energy scale which fixes the amplitude of the CMB anisotropies and $p$ is a free index. In this case the slow-roll trajectory is explicitly known and one can calculate $\phi_{*}$, the field vacuum expectation value at Hubble radius crossing from $\phi_{\mathrm{end}} / M_{\mathrm{P} l}=p / \sqrt{2}$, the field vacuum expectation value (VEV) at which inflation stops. One gets 27]

$$
\phi_{*}^{2}=2 p M_{\mathrm{P} l}^{2} \Delta N_{*}+\phi_{\mathrm{end}}^{2} .
$$

The reheating phase in large field models proceeds by parametric oscillations around the minimum of the potential and it is well known that the corresponding equation of state parameter is given by [6, 17, 18, 50]

$$
\bar{w}_{\mathrm{reh}}=\frac{p-2}{p+2} .
$$

In particular, for $p=2$, one obtains $\bar{w}_{\text {reh }}=0$ : that is to say the oscillatory phase is equivalent to a matter dominated era (the quartic case corresponding to a radiation dominated era, and so on). Although this formula is derived without taking into account the coupling between the inflaton field and radiation, we show in Appendix A that it is a very good approximation.

Knowing explicitly the equation during reheating, we are now in a position where the algebraic equations (19) and (20) can be solved exactly. After some algebra, one obtains

$$
\begin{aligned}
\Delta N_{*}^{\text {nuc }}= & -\frac{p}{4}-\frac{p^{2}-2 p+4}{12 p} \mathrm{~W}_{0}\left\{-\frac{12 p}{p^{2}-2 p+4}\right. \\
& \left.\times \exp \left[-\frac{12 p\left(\mathcal{N}^{\text {nuc }}+p / 4\right)}{p^{2}-2 p+4}\right]\right\},
\end{aligned}
$$

and

$$
\begin{aligned}
\Delta N_{*}^{\text {end }}= & -\frac{p}{4}-\frac{p-2}{8} \mathrm{~W}_{0}\left\{-\frac{8}{p-2}\right. \\
& \left.\times \exp \left[-\frac{8\left(\mathcal{N}^{\text {end }}+p / 4\right)}{p-2}\right]\right\},
\end{aligned}
$$

where $\mathrm{W}_{0}$ is a Lambert function. Both quantities $\mathcal{N}^{\text {nuc }}$ and $\mathcal{N}^{\text {end }}$ depend only on the model parameter $p$ and the amplitude of the observed anisotropies. Explicitly, $\mathcal{N}^{\text {nuc }}$ reads

$$
\begin{aligned}
\mathcal{N}^{\text {nuc }} & =-N_{0}+\frac{2}{3 p}(p-1) \ln \left(2 \pi \sqrt{120} \frac{Q_{\mathrm{rms}-\mathrm{PS}}}{T}\right) \\
& -\frac{p+2}{6 p} \ln \left[9 \frac{2^{\left(-p^{2}+p-6\right) /(p+2)}}{p^{\left(-p^{2}+2 p-4\right) /(2 p+4)}}\right]-\frac{p-4}{3 p} \ln \frac{\rho_{\mathrm{nuc}}}{M_{\mathrm{Pl}}},
\end{aligned}
$$

where the amplitude of the CMB anisotropies has been expressed in terms of the quadrupole moment

$$
\frac{Q_{\mathrm{rms}-\mathrm{PS}}}{T}=\sqrt{\frac{5 C_{2}}{4 \pi}} \simeq 6 \times 10^{-6} .
$$

In Eq. (26), the last term vanishes for $p=4$ since, as already noticed above, the phase of oscillations is equivalent to a radiation dominated era which cannot be distinguished from the subsequent hot big-bang epoch. On 
the other hand, the constant $\mathcal{N}^{\text {end }}$ can be expressed as

$$
\begin{aligned}
\mathcal{N}^{\text {end }}=-N_{0} & +\frac{1}{2} \ln \left(2 \pi \sqrt{120} \frac{Q_{\mathrm{rms}-\mathrm{PS}}}{T}\right) \\
& -\frac{1}{4} \ln \left(9 \frac{2^{1-p}}{p^{1-p / 2}}\right) .
\end{aligned}
$$

From Eqs. (24) and (25), we immediately deduce that, for the large field models, the range of allowed values of $\Delta N_{*}$ strongly depends on $p$. In Figs. 1 and 2 we have plotted the large field predictions, obtained from Eqs. (24) and (25), for the slow-roll parameters $\epsilon_{1}\left(\Delta N_{*}\right)$ and $\epsilon_{2}\left(\Delta N_{*}\right)$ compared to the one- and twosigma WMAP7 confidence intervals (see Appendix B). The first figure represents the slow-roll predictions in the plane $\left(n_{\mathrm{S}}, r\right)$, while the second one corresponds to the plane $\left(\epsilon_{1}, \epsilon_{2}\right)$. The annotated values trace the quantity $\log \left(g_{*}^{1 / 4} T_{\text {reh }} / \mathrm{GeV}\right)$, where the reheating temperature is defined by the relation

$$
g_{*}^{1 / 4} T_{\mathrm{reh}} \equiv\left(\frac{30}{\pi^{2}} \rho_{\mathrm{reh}}\right)^{1 / 4},
$$

and $g_{*}$ is the number of relativistic degrees of freedom at that time. Large values of $p$ cannot explain the current measurements of $n_{\mathrm{S}}$ and $r$, while the low values can. Therefore, it is clear that there now exists a lower bound on the reheating temperature. In Sec. III, we derive the Bayesian two-sigma limits on $\rho_{\text {reh }}$ (or $g_{*}^{1 / 4} T_{\text {reh }}$ ) by including the reheating parameter into the data analysis process. These plots should make evident that the reheating in the large field models is already observable with the current CMB data, and more than being a nuisance parameter, it is actually constrained.

Let us also remark that the case $p=4$ is particularly interesting; see the blue point annotated "16" in Figs. 1 and 2 Indeed, the value $p=4$ is the extreme case in which $\Delta N_{*}$ is actually fixed to

$$
\Delta N_{*}^{p=4}=-N_{0}+\frac{1}{2} \ln \left(2 \pi \sqrt{120} \frac{Q_{\mathrm{rms}-\mathrm{PS}}}{T}\right) \simeq 58.5 .
$$

This is why this model is represented by a single point in Figs. 1 and 2, Making any other choice is equivalent to assuming a more complicated reheating model which should at least be specified. For instance, Ref. [45] (see Fig. 19) uses two values, 50 and 60 e-folds, instead of one. From the above considerations, it is clear that 50 is much too small. But, of course, one can always assume that the shape of the potential in the slow-roll regime is not the same as in the reheating regime (actually, this has to be the case for small field models, see below). In this case $V(\phi) \propto \phi^{4}$ is not relevant during the oscillations of the field and $\bar{w}_{\text {reh }} \neq 1 / 3$. However, as discussed in the next section, even in this case, the reheating epoch is still constrained. The same range of variations for $\Delta N_{*}$ has also been used in Ref. [51] (see Fig. 2) for the $\phi^{2}$ model. Compared to our results, 50 is too high and excludes models which are still allowed while 60 predicts a reheating energy scale higher the energy scale at the end of inflation: $\rho_{\text {reh }}>\rho_{\text {end }}$.

\section{Small field models}

One of the reasons leading to such a strong reheating influence on the large field model predictions comes from Eq. (23). Once the potential is chosen, the spectral index and tensor-to-scalar ratio are intimately linked to the way the reheating proceeds. One may therefore wonder how the reheating can influence the model predictions in a case where it is unrelated to the shape of the primordial power spectra. As a motivated example, we discuss in this section the case of the small field models ending with a reheating characterized by a mean equation of state $\bar{w}_{\text {reh. }}$. The small field potential reads

$$
V(\phi)=M^{4}\left[1-\left(\frac{\phi}{\mu}\right)^{p}\right]
$$

where $\mu$ represents a VEV for the field $\phi, p$ a power index, and $M$ fixes the amplitude of the observed anisotropies. Inflation proceeds from small to large values of the field. For convenience, we denote by $\chi$ the field value in units of $\mu$, i.e. $\chi \equiv \phi / \mu$. The slow-roll trajectory in terms of $\chi$ reads [27]

$$
\Delta N_{*}=\frac{\mu^{2}}{2 p M_{\mathrm{Pl}}^{2}}\left(\chi_{*}^{2}+\frac{2}{p-2} \chi_{*}^{2-p}-\chi_{\mathrm{end}}^{2}+\frac{2}{2-p} \chi_{\mathrm{end}}^{2-p}\right),
$$

where, again, $\chi_{\text {end }}$ is defined by $\epsilon_{1}\left(\chi_{\text {end }}\right)=1$, i.e.

$$
\chi_{\mathrm{end}}^{p-1}=\frac{\sqrt{2}}{p} \frac{\mu}{M_{\mathrm{Pl}}}\left(1-\chi_{\mathrm{end}}^{p}\right) .
$$

Both of these equations do not have an explicit solution (unless $p=2$ ) but can be numerically solved for a given set of model parameters $\mu$ and $p$. For this reason, instead of deriving the reheating allowed values for $\Delta N_{*}$, it is more convenient to derive the reheating allowed values for $\chi_{*}$. In fact, $\chi_{*}$ should lie between $\chi_{*}^{\text {nuc }}$ and $\chi_{*}^{\text {end }}$, the field values such that reheating ends, respectively, at BBN and just after inflation. After some algebra, one finds $\chi_{*}^{\text {nuc }}$ to be the solution of

$$
\begin{aligned}
\frac{\mu^{2}}{2 p M_{\mathrm{Pl}}^{2}} & \left(\chi_{*}+\frac{2}{p-2} \chi_{*}^{2-p}\right)+\frac{3 \bar{w}_{\mathrm{reh}}}{3+3 \bar{w}_{\mathrm{reh}}} \ln \left(1-\chi_{*}^{p}\right) \\
& -\frac{3 \bar{w}_{\mathrm{reh}}+1}{3+3 \bar{w}_{\mathrm{reh}}} \ln \left(\chi_{*}^{p-1}\right) \\
& =\frac{\mu^{2}}{2 p M_{\mathrm{Pl}}^{2}}\left(\chi_{\text {end }}^{2}+\frac{2}{p-2} \chi_{\mathrm{end}}^{2-p}\right)+\mathcal{F}^{\mathrm{nuc}},
\end{aligned}
$$




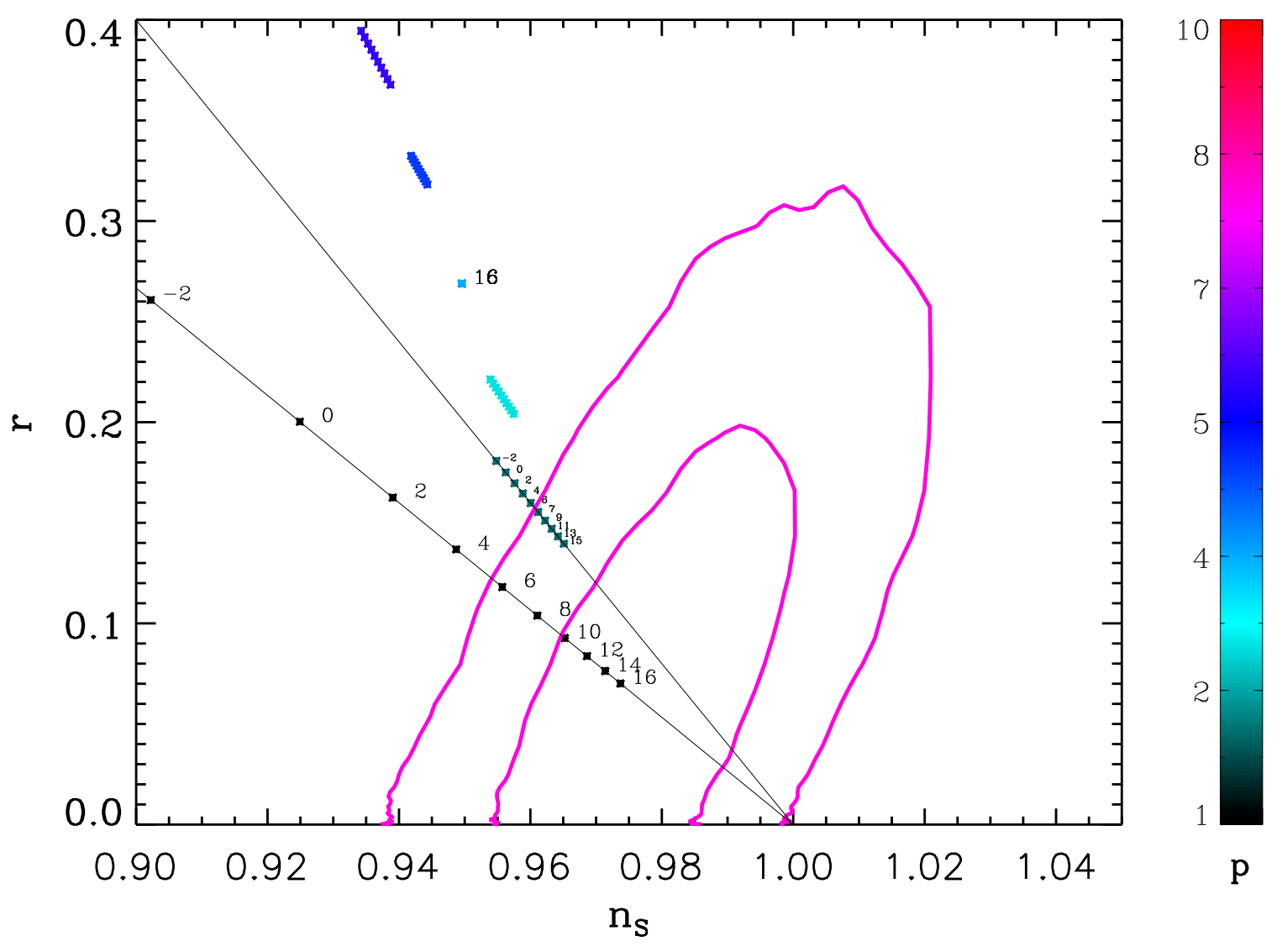

FIG. 1: Reheating consistent slow-roll predictions for the large field models in the plane $\left(n_{\mathrm{S}}, r\right)$. The two contours are the one and two-sigma WMAP confidence intervals (marginalized over second order slow-roll). The two lines represent the locus of the $p \gtrsim 1$ and $p=2$ models while the blue point annotated " 16 " corresponds to $p=4$. The annotations trace the energy scale at which the large field reheating ends and correspond to $\log \left(g_{*}^{1 / 4} T_{\text {reh }} / \mathrm{GeV}\right)$. Clearly, these values are limited from below to stay inside the two-sigma contours.

with

$$
\begin{aligned}
& \mathcal{F}^{\text {nuc }}=-N_{0}+\frac{1+3 \bar{w}_{\mathrm{reh}}}{3+3 \bar{w}_{\mathrm{reh}}} \ln \left(2 \pi \sqrt{120} \frac{Q_{\mathrm{rms}-\mathrm{PS}}}{T}\right) \\
& -\frac{1}{3+3 \bar{w}_{\mathrm{reh}}} \ln \left[9\left(\frac{\mu}{M_{\mathrm{Pl}} p}\right)^{3 \bar{w}_{\mathrm{reh}}+1} 2^{\left(3 \bar{w}_{\mathrm{reh}}-1\right) / 2}\right] \\
& -\frac{1}{3+3 \bar{w}_{\mathrm{reh}}} \ln \left(1-\chi_{\mathrm{end}}^{p}\right)+\frac{1-3 \bar{w}_{\mathrm{reh}}}{3+3 \bar{w}_{\mathrm{reh}}} \ln \left(\frac{\rho_{\mathrm{nuc}}^{1 / 4}}{M_{\mathrm{P} l}}\right) .
\end{aligned}
$$

Similarly, solving for $\rho_{\text {reh }}=\rho_{\text {end }}$ gives $\chi_{*}^{\text {end }}$ as the solution of

$$
\begin{aligned}
& \frac{\mu^{2}}{2 p M_{\mathrm{P} l}^{2}}\left(\chi_{*}^{2}+\frac{2}{p-2} \chi_{*}^{2-p}\right)+\frac{1}{4} \ln \left(1-\chi_{*}^{p}\right)-\frac{1}{2} \ln \left(\chi_{*}^{p-1}\right) \\
& =\frac{\mu^{2}}{2 p M_{\mathrm{P} l}^{2}}\left(\chi_{\mathrm{end}}^{2}+\frac{2}{p-2} \chi_{\mathrm{end}}^{2-p}\right)+\mathcal{F}^{\mathrm{end}},
\end{aligned}
$$

where

$$
\begin{aligned}
\mathcal{F}^{\mathrm{end}}=-N_{0} & +\frac{1}{2} \ln \left(2 \pi \sqrt{120} \frac{Q_{\mathrm{rms}-\mathrm{PS}}}{T}\right) \\
& -\frac{1}{4} \ln \left[9\left(\frac{\mu}{M_{\mathrm{Pl}} p}\right)^{2}\left(1-\chi_{\mathrm{end}}^{p}\right)\right] .
\end{aligned}
$$

As a result, for given values of $\mu, p$ and $\bar{w}_{\text {reh }}$, one has first to solve Eq. (33) to get $\chi_{\text {end }}$, then Eqs. (34) and (36) to obtain $\chi_{*}^{\text {nuc }}$ and $\chi_{*}^{\text {end }}$ from which $\Delta N_{*}^{\text {nuc }}$ and $\Delta N_{*}^{\text {end }}$ are deduced by using Eq. (32). From the value of $\chi_{*}$, one can also directly evaluate the two slow-roll parameters $\epsilon_{1 *}$ and $\epsilon_{2 *}$. Let us notice that some of the expressions above can be ill defined if $p=2$. In this case, Eqs. (34) and (36) should be rederived from the start and one can show that it then always leads to well-defined expressions. The rest is the same as for the large field models (see Sec. II C), $\rho_{\text {reh }}$ being in one-to-one correspondence with the value of $\Delta N_{*}$ through Eqs. (10) and (13) once a value of $\bar{w}_{\text {reh }}$ has been chosen. It is worth emphasizing again 


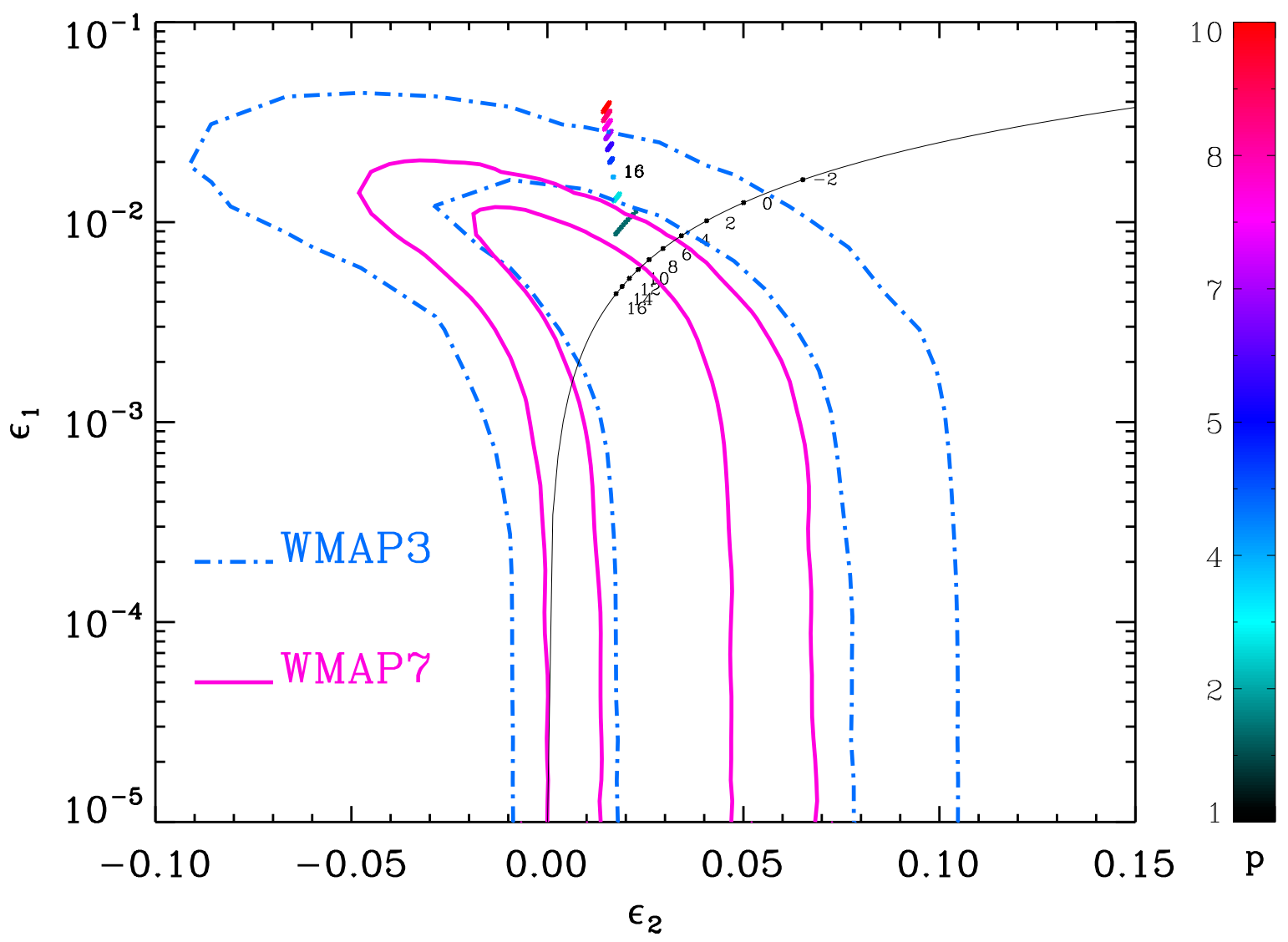

FIG. 2: Reheating consistent slow-roll predictions for the large field models in the plane $\left(\epsilon_{1}, \epsilon_{2}\right)$. The two blue dot-dashed contours are the one- and two-sigma WMAP3 confidence intervals (marginalized over second order slow-roll) while the pink solid contours are the one- and two-sigma WMAP7 ones. As in Fig. 1 the annotations trace the energy scale at which the large field reheating ends and correspond to $\log \left(g_{*}^{1 / 4} T_{\mathrm{reh}} / \mathrm{GeV}\right)$. The solid line represents the model $p \gtrsim 1$. This confirms that there now exists a lower bound on the value of $g_{*}^{1 / 4} T_{\text {reh }}$ (see Sec. III).

that, in order to derive these results, no assumption has been made about the reheating epoch which is entirely characterized by $\rho_{\text {reh }}$ and $\bar{w}_{\text {reh }}$.

In Fig. 3, we have represented the slow-roll predictions for an assumed generic value of $p=3$ and various values of $\bar{w}_{\text {reh }}$ ranging from -0.2 to 0.8 . The annotations are the values of $\log \left(g_{*}^{1 / 4} T_{\text {reh }} / \mathrm{GeV}\right)$ while the color scale traces the values of $\mu / M_{\mathrm{P} l}$. For small field models, the reheating energy scale is all the more so constrained that $\bar{w}_{\text {reh }}$ and $\mu$ are small. In fact, these plots show that $\bar{w}_{\text {reh }}$ and $\mu$ are degenerated: it is possible to render compatible a low value of $\bar{w}_{\text {reh }}$ provided $\mu$ is super-Planckian. Conversely, small values of $\mu$ can be made compatible with the data for a high energy scale reheating if $\bar{w}_{\text {reh }}<1 / 3$, or a low energy scale reheating for $\bar{w}_{\text {reh }}>1 / 3$. In the next section, we perform a full analysis of the small field models, reheating included, in view of the WMAP7 data to quantify the above claims in terms of posterior probability distributions.

\section{INFERRING REHEATING FROM CMB DATA}

In view of the previous results, the correct way to discuss how well the CMB data constrain a set of known inflationary models is to perform a Bayesian analysis of the data given the model parameters, including the reheating. Notice that this is different than constraining the slow-roll parameters, or the spectral index and tensorto-scalar ratio, which only encode the shape of the primordial power spectra and know nothing about reheating (whereas a model of inflation does).

\section{A. Exact numerical integration}

The numerical exact integration method has been introduced in Refs. [27, 47, 52, 53] and consists of the computation of the primordial power spectra assuming 

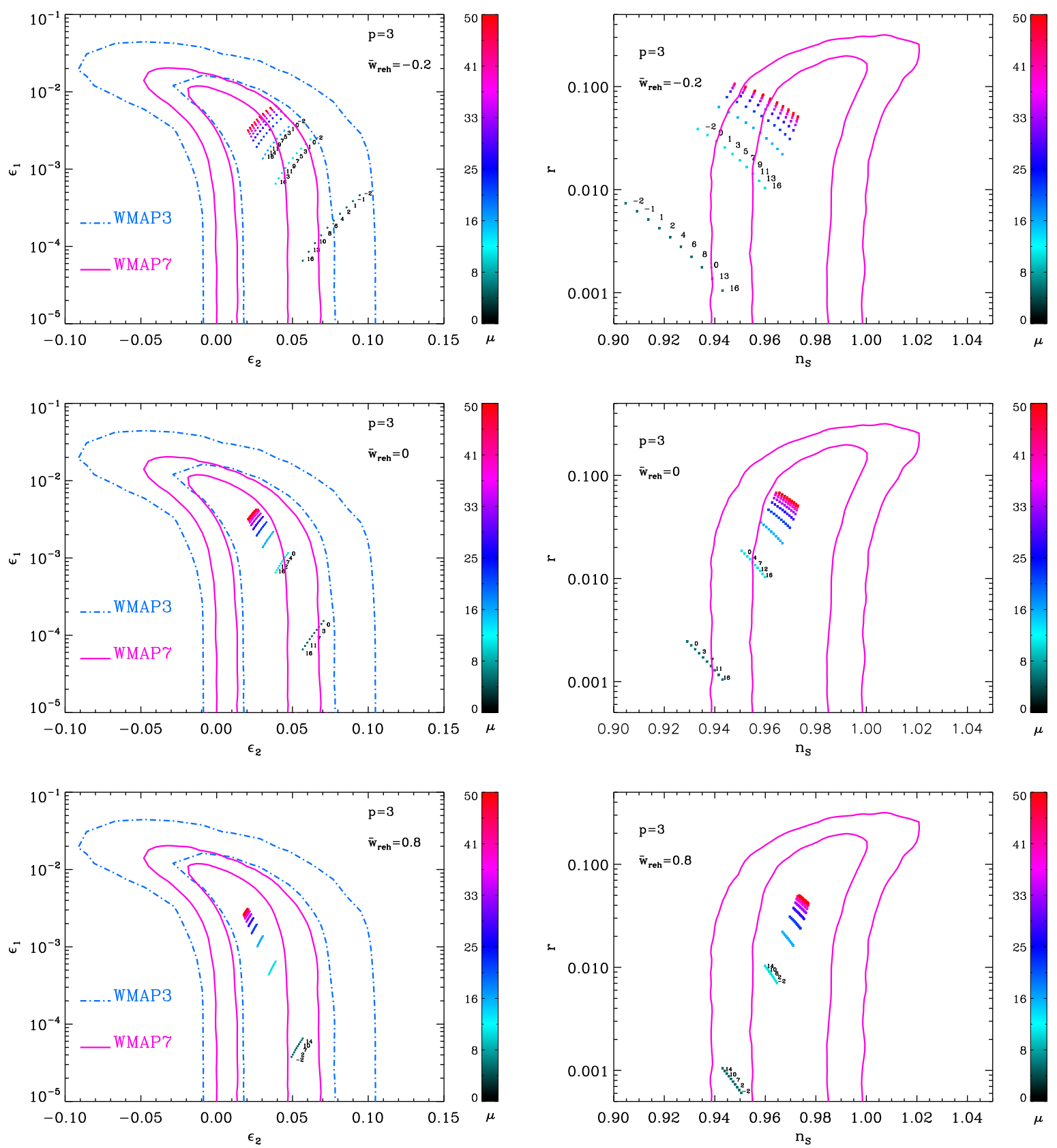

FIG. 3: Reheating consistent slow-roll predictions for the small field models with an assumed generic value $p=3$ and for $\bar{w}_{\text {reh }}=-0.2$ (top), $\bar{w}_{\text {reh }}=0$ (middle) and $\bar{w}_{\text {reh }}=0.8$ (bottom). The right panels display the corresponding predictions in the plane $\left(n_{\mathrm{S}}, r\right)$. The reheating has a strong influence for low values of both $\bar{w}_{\mathrm{reh}}$ and $\mu / M_{\mathrm{P} l}$. As in Figs. 1 and 2 the annotations give the values of $\log \left(g_{*}^{1 / 4} T_{\mathrm{reh}} / \mathrm{GeV}\right)$. It is also interesting to notice that the sequence of successive $T_{\text {reh }}$ is switched for $\bar{w}_{\text {reh }}=0.8$ (in fact for $\bar{w}_{\text {reh }}>1 / 3$ ), i.e. large reheating temperatures correspond to smaller spectral indices for $\bar{w}_{\text {reh }}=0.8$ (i.e. $\left.\bar{w}_{\text {reh }}>1 / 3\right)$ while, for $\bar{w}_{\text {reh }}=0$ or $\bar{w}_{\text {reh }}=-0.2\left(\right.$ i.e. $\left.\bar{w}_{\text {reh }}<1 / 3\right)$, they correspond to larger $n_{\mathrm{S}}$.

only General Relativity and linear perturbation theory. Therefore, the only model parameters are the ones appearing in the inflaton potential together with the reheating parameter $R_{\mathrm{rad}}$, for the very reasons explained in Sec. [II] The numerical integration of the inflationary perturbations sets up the initial conditions for the subsequent cosmological perturbations from which the CMB anisotropies are deduced. For this purpose, we have used a modified version of the CAMB code [54] coupled to a Monte-Carlo-Markov-Chain (MCMC) exploration of the parameter space implemented in the COSMOMC code [55] and given the WMAP7 data 44-46]. Con- 
cerning the standard cosmological model, we have assumed a flat $\Lambda \mathrm{CDM}$ model having five parameters: the density parameter of baryons, $\Omega_{\mathrm{b}}$, of cold dark matter $\Omega_{\mathrm{dm}}$, the Hubble parameter today $H_{0}$, the optical depth $\tau$ encoding the redshift at which the Universe reionized, and the nuisance parameter $A_{\mathrm{Sz}}$ encoding the relative amplitude of the diffuse Sunyaev-Zel'dovich (SZ) effect compared to the analytical model of Ref. [56]. In fact, as discussed in Ref. [55], it is more convenient to sample the cosmological parameter space along the rescaled quantity $\left(\Omega_{\mathrm{b}} h^{2}, \Omega_{\mathrm{dm}} h^{2}, \tau, \theta, A_{\mathrm{Sz}}\right)$ where $H_{0}=$ $100 \mathrm{hm} / \mathrm{s} / \mathrm{Mpc}$ and $\theta$ measures the ratio of the sound horizon at last scattering to the angular diameter distance. Following Ref. [45], we have included the lensing corrections on the temperature and polarization power spectra, and, to limit parameter degeneracies, completed the WMAP7 data with the latest Hubble Space Telescope (HST) bound on $H_{0}$ [57]. Concerning the primordial parameters, they are now provided by our inflationary model parameters, up to some observationally convenient rescaling. For instance, we will prefer to sample on $P_{*}$, the amplitude of the scalar perturbation at the pivot scale, rather than on the potential normalization $M$, both being in one-to-one correspondence. Similarly, it is more convenient to sample the reheating era over the parameter $R$,

$$
R \equiv R_{\mathrm{rad}} \frac{\rho_{\mathrm{end}}^{1 / 4}}{M_{\mathrm{P} l}}
$$

rather than $R_{\text {rad }}$. As can be seen by comparing Eq. (15) and the following exact expression:

$$
\ln R=N_{\mathrm{T}}-N_{*}+N_{0}+\frac{1}{2} \ln \left(3 \frac{V_{\text {end }}}{V_{*}} \frac{3-\epsilon_{1 *}}{3-\epsilon_{1 \mathrm{end}}}\right),
$$

contrary to $R$, the values of $R_{\text {rad }}$ explicitly depend on $P_{*}$. This would induce unwanted correlations between $R_{\text {rad }}$ and $P_{*}$ which are therefore avoided by sampling the reheating over $R$. Notice that since $R$ and $R_{\text {rad }}$ differ by a factor $\rho_{\text {end }}$, they are also in one-to-one correspondence once the model of inflation is specified [27]. In order to perform the MCMC analysis, we still have to specify the prior probability distributions. Concerning the cosmological parameters, we have chosen wide flat priors around the preferred posterior values obtained by the WMAP team [45]. The reheating energy scale being unknown, we assume a flat prior on $\ln R$ whose extension is given by the consistency conditions mentioned in Sec. II B. Reheating should occur before nucleosynthesis and after the end of inflation while the positivity energy conditions imply $-1 / 3<\bar{w}_{\text {reh }}<1$. As a result, we take a flat prior for $\ln R$ in the range [27]

$$
\ln \left(\frac{\rho_{\mathrm{nuc}}^{1 / 4}}{M_{\mathrm{P} l}}\right)<\ln R<-\frac{1}{3} \ln \left(\frac{\rho_{\mathrm{nuc}}^{1 / 4}}{M_{\mathrm{P} l}}\right)+\frac{4}{3} \ln \left(\frac{\rho_{\mathrm{end}}^{1 / 4}}{M_{\mathrm{P} l}}\right) .
$$

The lower bound is approximately $\simeq-47$ for $\rho_{\text {nuc }}=$ $10 \mathrm{MeV}$ whereas the upper bound depends on $\rho_{\text {end }}$, and thus on the other inflationary model parameters. Finally, we have chosen a flat prior on the logarithm of $P_{*}$ around the value giving the right amplitude of the CMB anisotropies: $2.7<\ln \left(10^{10} P_{*}\right)<4.0$. Let us notice that since $P_{*}$ is well constrained, this translates into an upper bound on $\ln \left(\rho_{\text {end }}^{1 / 4} / M_{\mathrm{Pl}}\right)<-5.5$ (analogous to the upper bound on $H_{*}$ in the slow-roll approximation) that will therefore be inherited by $\ln R$ so that the maximal value of the upper bound is $\simeq 8.3$. The other prior choices on the primordial parameters are those concerning the inflaton potential and will be specified later.

In the following, we perform the WMAP7 data analysis along those lines for both the large and small field models. As the first step, we sample over the rescaled reheating parameter $\ln R$ without any assumptions on $\bar{w}_{\text {reh }}$. We show that it is actually constrained for all models. As can be checked in Eq. (10), it means that the CMB data restrict the a priori possible values of $\Delta N$ and $\bar{w}_{\text {reh }}$. Conversely, this result shows that not including the reheating parameter when constraining inflationary models is no longer a reasonable option. For the second step, we break the degeneracy between $\bar{w}_{\text {reh }}$ and $\Delta N$ and assume that $\bar{w}_{\text {reh }}$ takes its natural values for the large field models given in Eq. (23), or choose a specific value in the small field models. These reasonable extra assumptions translate the bounds on $\ln R$ into a lower limit on $\rho_{\text {reh }}$ and/or $g_{*}^{1 / 4} T_{\text {reh. }}$. Unless specified, we have stopped the MCMC exploration according to the $\mathrm{R}$-statistics 58 . implemented in COSMOMC such that the difference in variances between the different Markov chains does not exceed a few percent. Typically, this corresponds to a set of 300000 to 500000 samples depending on the underlying model of inflation.

\section{B. Large field models}

The potential for the large field models is given in Eq. (21). Together with $P_{*}$ and $\ln R$, there is only one additional primordial parameter $p$ for which we have chosen a flat prior in the range $p \in[0.2,5]$. The upper bound is motivated by the previous constraints on large fields [27] whereas the lower one is a theoretical prejudice associated with the non-naturalness of extremely small values of $p$ in any field theory. The marginalized posterior distributions for the sampled and derived cosmological parameters are represented in Fig. 4 for the two prior assumptions detailed in the following. The solid lines are without any assumption on the reheating whereas the dashed ones are under the natural equation of state $\bar{w}_{\text {reh }}=(p-2) /(p+2)$. These probabilities are compatible with the one already derived in the literature [45, 59] up to slight shifts coming from changing the reheating assumptions. This is the result of some tension between the large field models which generically predict a large tensor-to-scalar ratio $r$ and its non-observation. Being more restrictive on the reheating gives less flexibility to the model such that $H_{0}$ and $\Omega_{\mathrm{b}}$ are slightly shifted to compensate for the too high $r$ values. 

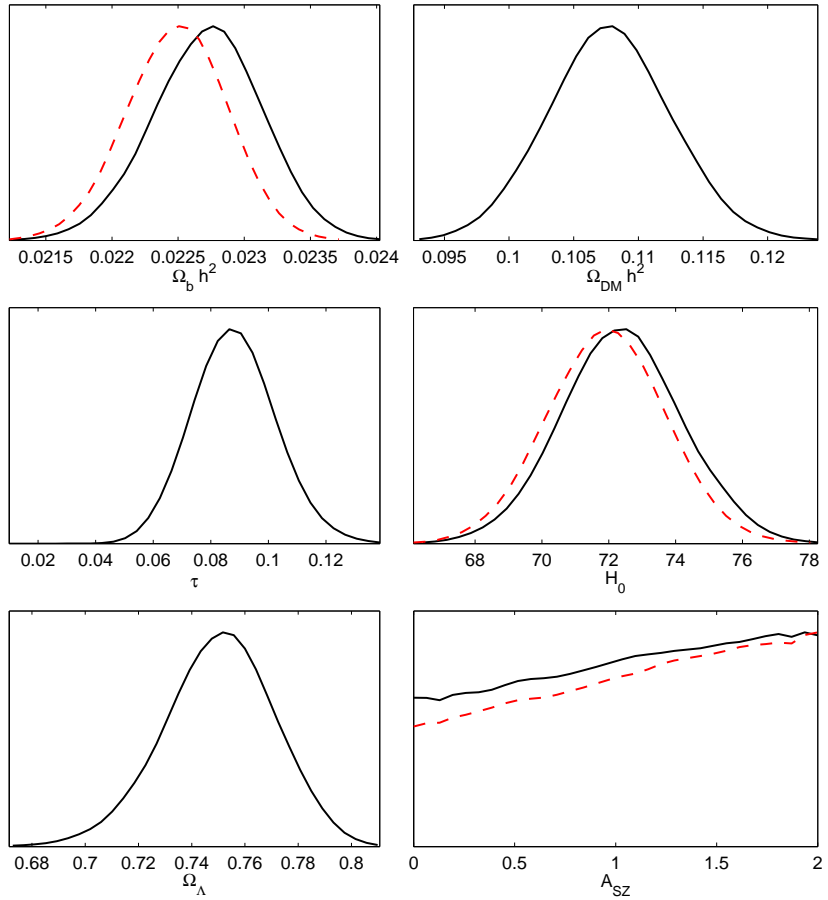

FIG. 4: Marginalized posterior probability distributions for the base and derived cosmological parameters in large field inflation. The black solid lines are without any assumptions on the large field reheating whereas the red dashed ones are under the prior $\bar{w}_{\text {reh }}=(p-2) /(p+2)$. They have been represented only when they differ with respect to the former.
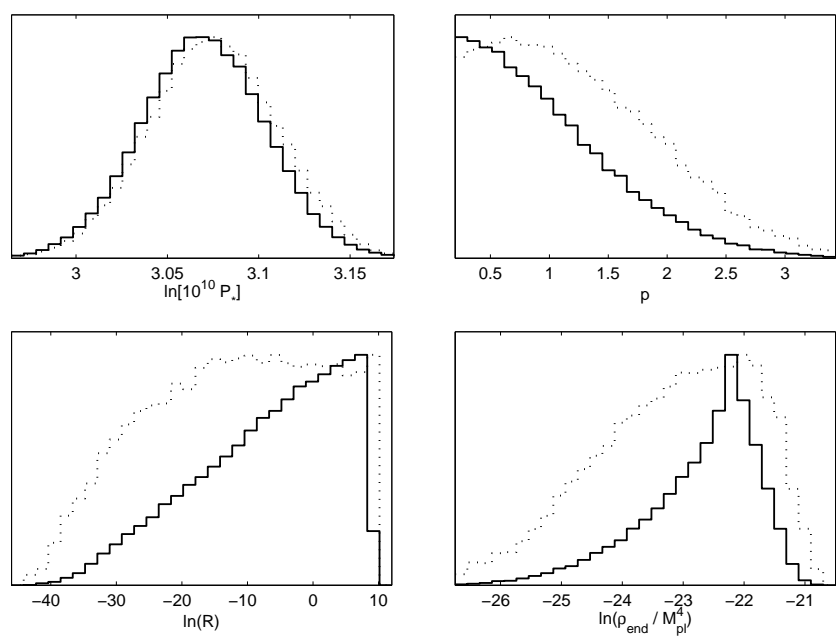

FIG. 5: Marginalized posterior probability distributions (solid lines) and mean likelihoods (dotted) for the large field model primordial parameters. This is without assumption on the reheating era. Notice the lower bound on the reheating parameter $\ln R$ which correlates with the potential power $p$ (see also Fig. 6). The energy scale at the end of large field inflation is also constrained.

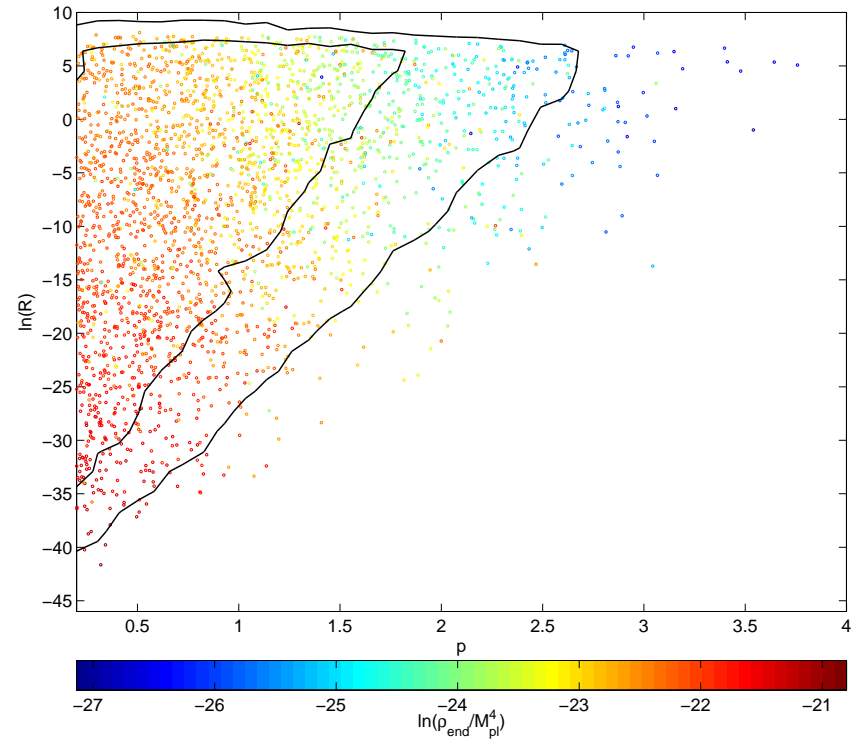

FIG. 6: Two-dimensional marginalized posterior probability distribution (point density) in the plane $(p, \ln R)$ and its oneand two-sigma confidence intervals. Correlations with the energy scale of large field inflation are traced by the color scale.

In Fig. 5, we have plotted the marginalized probability distribution for the large field primordial parameters without assumption on the reheating. It is particularly interesting to compare these plots to Fig. 18 of Ref. 27] since this allows us to see the improvements on the parameter constraints coming from the passage from WMAP3 data to WMAP7. In addition to the expected constraints on $P_{*}$, we find the $95 \%$ confidence limit

$$
p<2.2,
$$

suggesting that $\phi^{2}$ inflation may now be considered under pressure. Let us emphasize that this result is robust against any possible reheating evolution since marginalized over $\ln R$. Concerning this last parameter, we find a 95\% lower bound:

$$
\ln R>-28.9 \text {. }
$$

In fact, as can be checked in Fig. 6, these two parameters are correlated together and also with $\rho_{\text {end }}$. These correlations can be understood as follows. From Eq. (39), the quantities $\ln R, p$ and $\ln \left(\rho_{\mathrm{end}} / M_{\mathrm{P} l}^{4}\right)$ are related by the formula

$$
\begin{aligned}
\ln \left(\frac{\rho_{\mathrm{end}}}{M_{\mathrm{P} l}^{4}}\right)= & \ln \left(128 \pi^{2} P_{*}\right)-2 N_{0}+2 \ln R-\frac{2}{1-n_{\mathrm{S}}} \\
& -\frac{p}{2} \frac{1+n_{\mathrm{S}}}{1-n_{\mathrm{S}}}+\ln \left[\frac{8 p\left(1-n_{\mathrm{S}}\right)}{p+2}\right],
\end{aligned}
$$

where $P_{*}$ and $n_{\mathrm{S}}$ are well constrained quantities. As a result, at fixed $\ln R$, the larger the $p$ values, the lower the energy scale at the end of inflation has to be, which is 

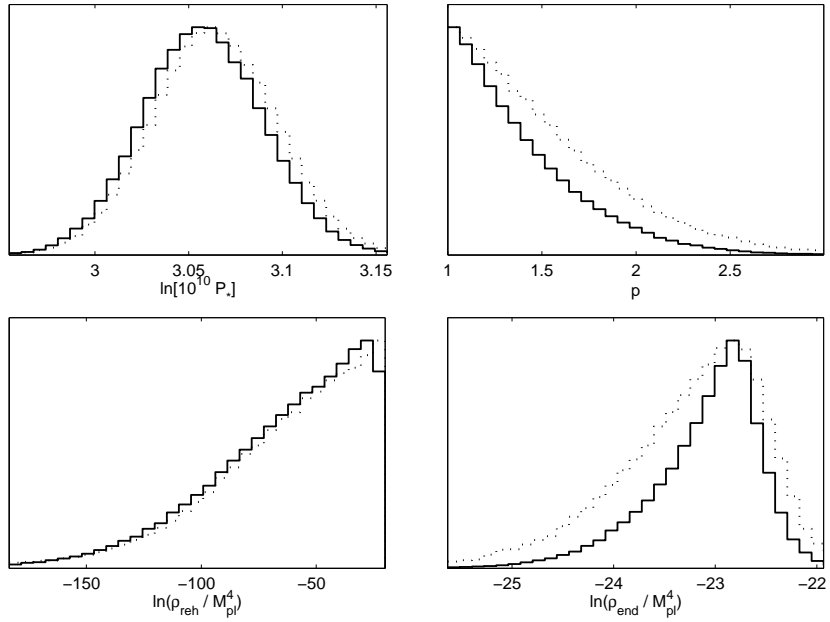

FIG. 7: Marginalized posterior probability distributions (solid lines) and mean likelihood (dotted lines) for the large field parameters when $\bar{w}_{\text {reh }}=(p-2) /(p+2)$. This extra-assumption on reheating yields to tighter constraints than in Fig. 5. In particular, we find $\rho_{\text {reh }}>17.3 \mathrm{TeV}$ at $95 \%$ confidence level, as well as $p<2.1$.

exactly what is observed in Fig. 6. Of course, $p$ cannot be too large since, in this case, the tensor-to-scalar ratio $r$ increases and rapidly becomes incompatible with the CMB data. In Fig. 6, we also observe that the smaller $p$, the larger the allowed range of variation of $\ln R$. The upper limit on $\ln R$ does not depend on $p$ and just comes from the upper limit on the energy scale of inflation ( $\ln R \lesssim 8.3$ ). On the other hand, the lower limit strongly depends on $p$ and represents a non trivial result. This expresses the fact that, for a given $p$, there are values of $\ln R$ for which there is no way to obtain, at the same time, a consistent reheating epoch and CMB predictions compatible with the data. From this effect, we also get the energy scale of large field inflation, and at two-sigma level

$$
4.4 \times 10^{15} \mathrm{GeV}<\rho_{\text {end }}^{1 / 4}<1.2 \times 10^{16} \mathrm{GeV} .
$$

The upper limit just comes from the constrain on the energy scale of inflation while the lower limit originates from the fact that $p$ cannot be too large (recall low values of $\rho_{\text {end }}$ means large value of $p$ ).

Assuming now that the reheating proceeds according to Eq. (23), one obtains the marginalized posteriors plotted in Fig. 7] and Fig. 8, To be consistent, we have modified our prior on $p$ by assuming a flat distribution in $p \in] 1,5]$; the case $p=1$ is a limiting case that may be problematic. Indeed, values of $p<1$ would induce $\bar{w}_{\text {reh }}<-1 / 3$ and inflation would not stop. Moreover, instead of using $\ln R$, we have used Eqs. (13) and (38) to sample the parameter space over $\ln \left(\rho_{\text {reh }} / M_{\mathrm{Pl}}^{4}\right)$ and from a flat prior in $\left[\ln \left(\rho_{\text {nuc }} / M_{\mathrm{Pl} l}^{4}\right), \ln \left(\rho_{\mathrm{end}} / M_{\mathrm{Pl} l}^{4}\right)\right]$. The upper bound on the $p$ posterior is slightly tighter due to the re-

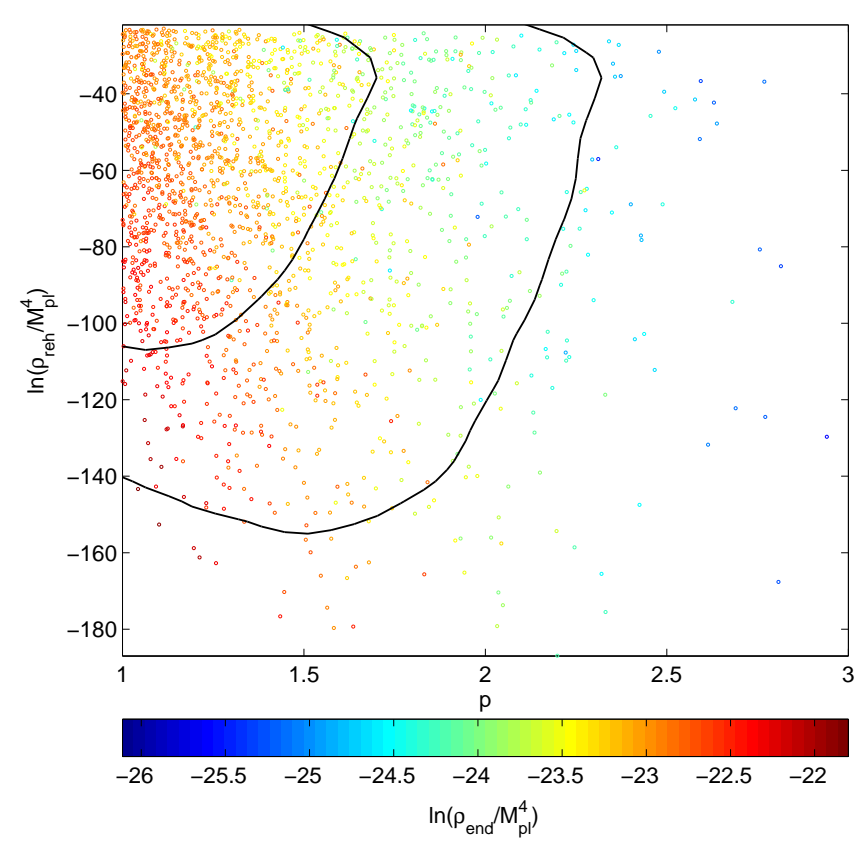

FIG. 8: One- and two-sigma marginalized limits in the plane $\left[p, \ln \left(\rho_{\text {reh }} / M_{\mathrm{Pl} l}^{4}\right)\right]$ for the large field models with $\bar{w}_{\text {reh }}=(p-$ $2) /(p+2)$. The point density traces the associated twodimensional posterior while the color map shows correlations with the energy scale at which inflation ends.

striction made over the reheating: we find the two-sigma limit $p<2.1$. For the same reasons, the energy scale of large field inflation is a bit more constrained, and the two-sigma range becomes

$$
5.2 \times 10^{15} \mathrm{GeV}<\rho_{\text {end }}^{1 / 4}<9.1 \times 10^{15} \mathrm{GeV} .
$$

Certainly, the more interesting result is the lower bound on the reheating energy scale. At $95 \%$ of the confidence limit

$$
\rho_{\mathrm{reh}}^{1 / 4}>17.3 \mathrm{TeV}
$$

The correlations between these three parameters are represented in Fig. 8 and have the same origin as the ones displayed in Fig. 6, up to the change of variable $R$ to $\rho_{\text {reh }}$. In particular, we see that, at a fixed value of $p$, the constraints on $\rho_{\text {reh }}$ are tighter for $p \lesssim 1.5$ than for $p \simeq 1.5$. This comes from the fact that the reheating is well constrained for a negative mean equation of state, which precisely corresponds to $p<2$ [see Eq. (23)]. The change of behavior around $p=1.5$ comes from this effect combined with a two high tensor-to-scalar ratio when $p \gtrsim 2$. Let us also emphasize that Eq. (46) is marginalized over all large field models. Coming with a theoretical preference for a given value of $p$ can lead to stronger bounds, as for instance if $p \gtrsim 1$ or $p=2$ (see Fig. 8). Finally, one can check that the bounds found in this section are compatible with the expectations we have derived from the slow-roll predictions of Sec. II C. 

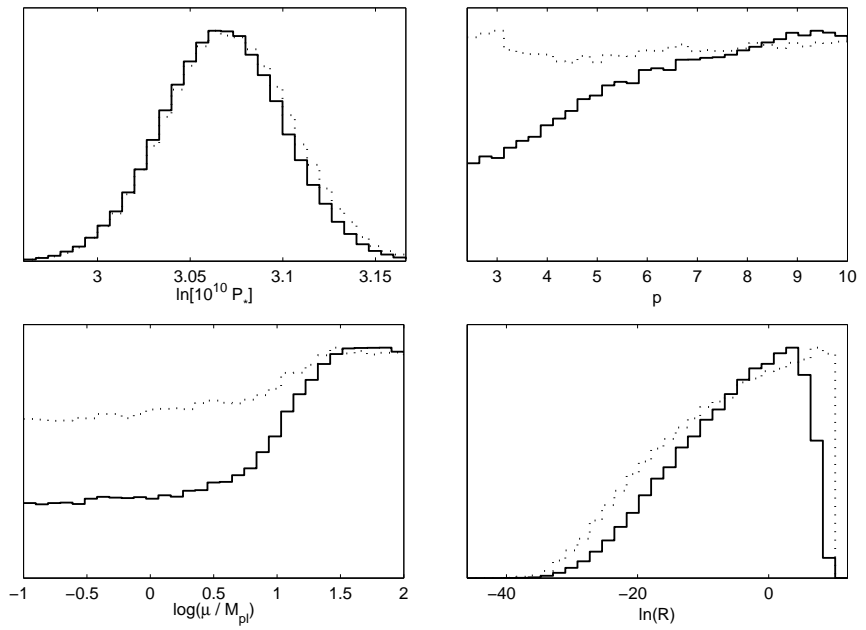

FIG. 9: Marginalized posterior probability distributions (solid lines) and mean likelihoods (dotted) for the small field model primordial parameters. This is without assumption on the reheating era. Notice again the lower bound on the reheating parameter $\ln R$ and the slightly favored super-Planckian values of $\mu$. Correlations are displayed in Fig. 10 .

In the next section, we perform a similar analysis for the small field models.

\section{Small field models}

The small field model potential of Eq. (31) involves an extra parameter compared to large fields which is the VEV $\mu$. The scale of this parameter being unknown, we have chosen a flat prior on $\log \left(\mu / M_{\mathrm{Pl}}\right)$ in the range $[-1,2]$. With the lower and upper limits being chosen only for numerical convenience, one should keep in mind that the physical values of $\mu$ may be larger or smaller. The important point is however that such a prior includes both sub-Planckian and super-Planckian values without prejudice. Concerning the potential index $p$, we have chosen a flat prior in the range $p \in[2.4,10]$. The upper bound is arbitrary whereas the lower bound excludes $p=2$ since this model is a special case [27]. In fact, there exists another reason that justifies the above choices. In the limit $\mu / M_{\mathrm{Pl}} \gg 1$, one can show, using a perturbative expansion in $M_{\mathrm{Pl}} / \mu$, that the two first horizon flow functions $\epsilon_{1}$ and $\epsilon_{2}$ become independent from $\mu$ and $p$, namely $\epsilon_{1}=\left(4 \Delta N_{*}+1\right)^{-1}$ and $\epsilon_{2}=4 \epsilon_{1}$. Therefore, it would be useless to take a larger upper bound on the $\mu$ prior since the corresponding physical predictions are no longer affected by this choice. All the other priors, both on the cosmological and primordial parameters, have been chosen as for the large field exploration. For the sake of clarity, we have not represented the marginalized posteriors for the cosmological parameters. Contrary to the large field models, these posteriors are almost the same whatever we assume for the reheating. The reason is that small field models do not have a tendency to produce a high tensor-to-scalar ratio. There is therefore no need for the cosmological parameters to compensate for such an effect and they decouple from the details of the inflationary and reheating phases. Finally, the cosmological parameter posteriors in small field inflation end up being very similar to the dashed curves (or solid curves when they are absent) plotted in Fig. 5 .

In Fig. 9 and Fig. 10, we have plotted the marginalized posterior probability distributions (one- and twodimensional, respectively) for the primordial small field parameters without assumption on the reheating. Again, we find the WMAP data to give a lower limit on the reheating parameter, at two-sigma level

$$
\ln R>-23.1
$$

Concerning the parameters $\mu$ and $p$, they are not constrained. However, the posteriors of Fig. 9 clearly show a tendency to favor super-Planckian values of $\mu$ together with large values of $p$. As can be seen in Fig. 10, since $\mu$ can take arbitrarily low values, the energy scale of small field inflation is not constrained from below. We find only the consistency condition that $\rho_{\text {end }}^{1 / 4}<9 \times 10^{15} \mathrm{GeV}$ from the $P_{*}$ limits. The correlations between $\mu$ and $p$ can be understood from Sec. $\amalg D$ and come from the requirements of having the right spectral index. The bound of Eq. (47) has the same origin but through the selection of the favored $\Delta N_{*}$ values. More details on these effects can be found in Refs. [27, 47].

As we did for large fields, we now assume an equation of state parameter for the small field reheating. Contrary to large field models, we no longer have a relationship between $\bar{w}_{\text {reh }}$ and the inflationary potential and one may only assume fiducial values ranging from $-1 / 3$ to 1. Again, the MCMC has been now sampled directly over $\ln \left(\rho_{\mathrm{reh}} / M_{\mathrm{pl}}^{4}\right)$ rather than over $\ln R$ by making use of Eqs. (13) and (38). The resulting one-dimensional posteriors for the primordial parameters have been plotted in Fig. 11 for the four values $\bar{w}_{\text {reh }}=-0.3,-0.2,-0.1$ and $\bar{w}_{\text {reh }}=0$, and only when the posteriors are affected by this choice. Comparing Figs. 9] and Fig. 11 shows that the posteriors of $\mu, p$ (and $P_{*}$ ) are mostly independent of the details of the reheating. The only quantity changing accordingly is $\rho_{\text {reh. }}$. This is not surprising since at a given $R$, changing the values of $\bar{w}_{\text {reh }}$ modifies the number of efolds the Universe reheated. As a result, the constraint on $R$ for small fields translates into a lower bound on $\rho_{\text {reh }}$ but only when $\bar{w}_{\text {reh }}$ is small. At $95 \%$ of confidence, we find

$$
\begin{array}{lll}
\bar{w}_{\text {reh }}=-0.3 & \Rightarrow & \rho_{\text {reh }}^{1 / 4}>8.9 \times 10^{5} \mathrm{GeV} \\
\bar{w}_{\text {reh }}=-0.2 & \Rightarrow & \rho_{\text {reh }}^{1 / 4}>3.9 \times 10^{2} \mathrm{GeV}
\end{array}
$$

while higher values of $\bar{w}_{\text {reh }}$ do not constrain $\rho_{\text {reh }}$ more than the prior $\rho_{\text {reh }}>\rho_{\text {nuc }}$. Physically, these results can be fully understood from Fig. 3. In particular, the 

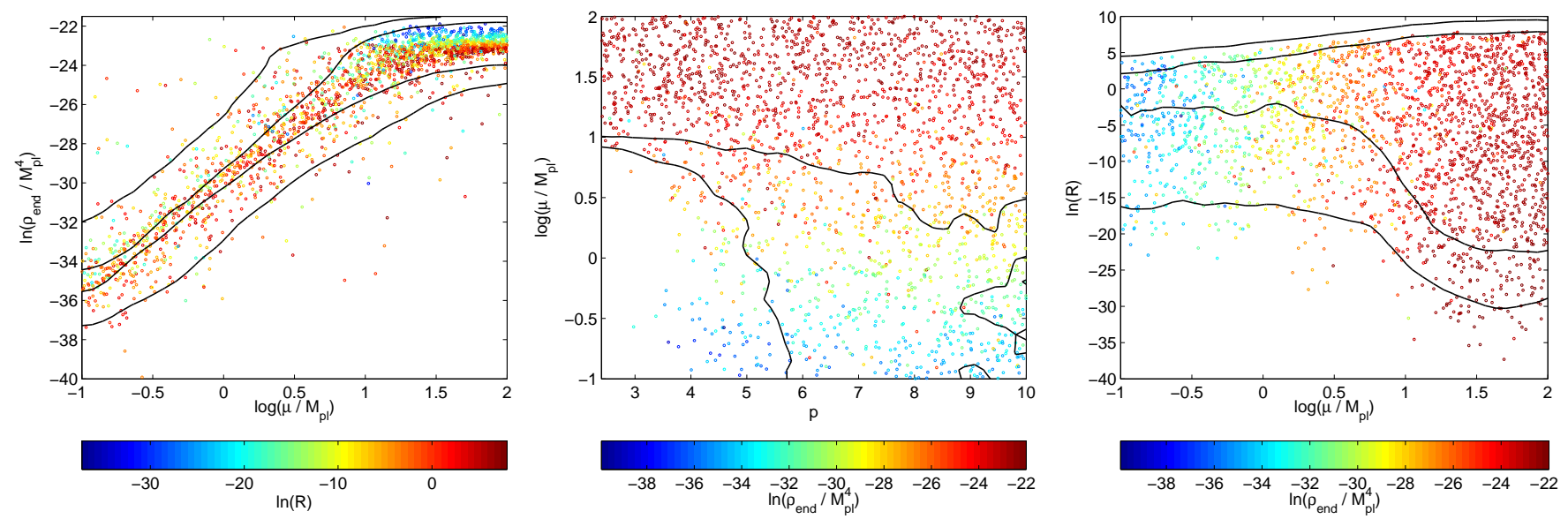

FIG. 10: One- and two-sigma contours of the two-dimensional marginalized probability distributions for small field inflation. The point density traces the associated two-dimensional posterior while the color scale shows correlations with third parameters. Notice the correlation between $\mu$ and $p$, as well as with $\ln R$.
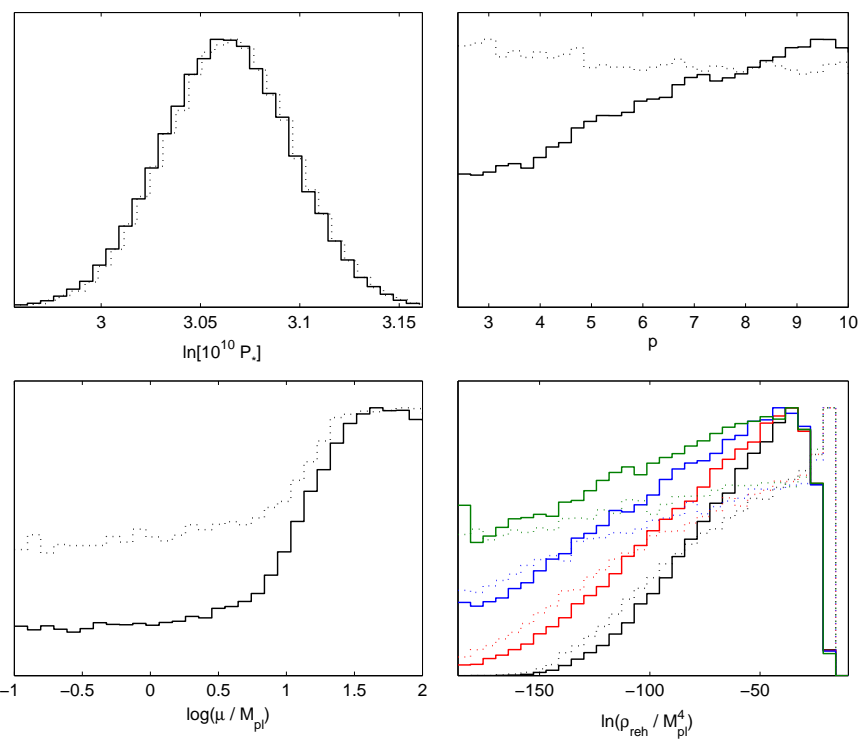

FIG. 11: Marginalized posterior probability distributions (solid lines) and mean likelihoods (dotted lines) for the small field model. These posteriors assume a constant equation of state with (from bottom to top) $\bar{w}_{\text {reh }}=-0.3$ (black lines), -0.2 (red lines), -0.1 (blue lines) and $\bar{w}_{\text {reh }}=0$ (green lines). Only $\rho_{\text {reh }}$ is affected by such prior choices (bottom right panel). The energy scale of reheating is all the more constrained from below than $\bar{w}_{\text {reh }}$ is small.

fact that constraints on $\rho_{\text {reh }}$ can be derived for negative values of $\bar{w}_{\text {reh }}$ is apparent from these plots. As expected, correlations between $\rho_{\text {reh }}$ and the other parameters are similar to the ones associated with $\ln R$. In Fig. 12, we have represented the two-dimensional posterior and its one- and two-sigma contours in the plane

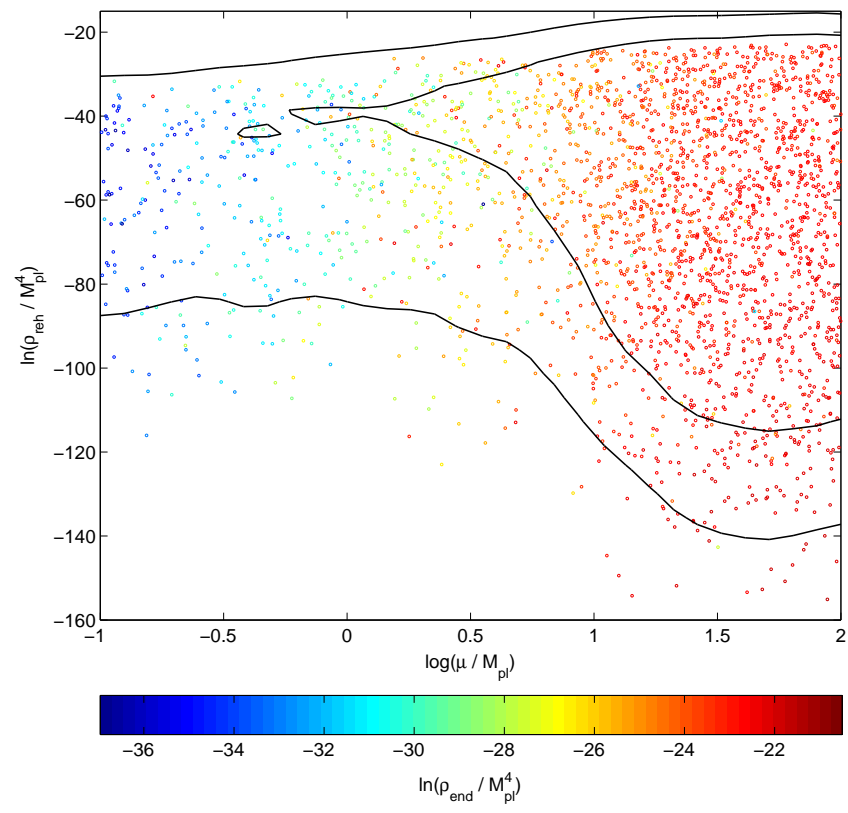

FIG. 12: One and two-sigma marginalized limits in the plane $\left[\log \left(\mu / M_{\mathrm{Pl}}\right), \ln \left(\rho_{\mathrm{reh}} / M_{\mathrm{Pl}}^{4}\right)\right]$ for the large field models with $\bar{w}_{\text {reh }}=-0.3$. The point density traces the associated twodimensional posterior while the color map shows correlations with the energy scale at which inflation ends.

$\left[\log \left(\mu / M_{\mathrm{P} l}\right), \ln \left(\rho_{\mathrm{reh}} / M_{\mathrm{P} l}^{4}\right)\right]$. The color scale traces correlations with the energy scale of small field inflation. We recover the slightly disfavored values of sub-Planckian vacuum expectation values. They clearly remain acceptable but only if the reheating ends at a high energy. Again, the previous results are compatible and easily understandable with the slow-roll predictions of Sec. IID. 
To conclude this section, we have found that the current WMAP data give non-trivial information on the reheating era in the two most considered classes of prototypical inflationary models (large and small fields). Conversely, when the goal is to use CMB data to constrain these models, including the reheating into the marginalization is definitely no longer an option.

\section{Importance sampling from slow-roll bounds}

The formulas derived in Sec. IIB link the reheating and inflationary model parameters to the spectral index and tensor-to-scalar ratio. As a result, they could be used to extract constraints on the reheating energy scale from some already derived constraints on the slow-roll parameters by using importance sampling [55]. However, this method is highly inefficient since most of the favored $\left(n_{\mathrm{S}}, r\right)$ values do not necessarily correspond to a consistent reheating model in a given inflationary framework. However, it clearly illustrates how knowledge on the primordial power spectra shape, complete with an inflaton potential, translates into some information on the energy scale at which the reheating ends. For this reason, we briefly discuss this method in the following although we prefer an exact numerical integration as performed above.

Assuming an inflationary model, with a known potential $V(\phi)$, one can generate any spectral index and tensorto-scalar ratio such that, at leading order in slow-roll,

$$
r=16 \epsilon_{1 *}, \quad n_{\mathrm{S}}-1=-2 \epsilon_{1 *}-\epsilon_{2 *},
$$

with

$$
\epsilon_{1 *}=\frac{1}{2}\left(\frac{V_{*}^{\prime}}{V_{*}}\right)^{2}, \quad \epsilon_{2 *}=-2\left[\frac{V_{*}^{\prime \prime}}{V_{*}}-\left(\frac{V_{*}^{\prime}}{V_{*}}\right)^{2}\right] .
$$

A prime here is understood as a derivative with respect to the field $\phi$. The star still refers to the time at which the scale under consideration crossed out the Hubble radius during inflation. Inverting Eq. (49), or Eq. (50), gives the value of $\phi_{*}$ (and eventually other parameters) leading to the required couple $\left(n_{\mathrm{S}}, r\right)$. For instance, in the large field models, one would find

$$
\frac{\phi_{*}}{M_{\mathrm{Pl}}}=\sqrt{\frac{8 \epsilon_{1 *}}{\epsilon_{2 *}^{2}}}, \quad p=4 \frac{\epsilon_{1 *}}{\epsilon_{2 *}} .
$$

From $\phi_{*}$ one can derive $\Delta N_{*}$ from the slow-roll trajectory and the value at which inflation stops, $\phi_{\text {end }}$. For instance, for large field inflation, one would obtain

$$
\Delta N_{*}=\frac{1-\epsilon_{1 *}}{\epsilon_{2 *}} .
$$

The energy scale at which reheating ends stems from
Eqs. (10) and (13):

$$
\begin{aligned}
\ln \left(\frac{\rho_{\mathrm{reh}}^{1 / 4}}{M_{\mathrm{Pl}}}\right) & =\frac{3+3 \bar{w}_{\mathrm{reh}}}{1-3 \bar{w}_{\mathrm{reh}}}\left(N_{0}+\Delta N_{*}\right) \\
& -\frac{1+3 \bar{w}_{\mathrm{reh}}}{2\left(1-3 \bar{w}_{\mathrm{reh}}\right)} \ln \left(8 \pi^{2} P_{*}\right)+\ln \sqrt{\epsilon_{1 *}} \\
& +\frac{1}{1-3 \bar{w}_{\mathrm{reh}}} \ln \left(\frac{3}{\epsilon_{1 *}} \frac{3-\epsilon_{1 *}}{3-\epsilon_{1 \mathrm{end}}} \frac{V_{\mathrm{end}}}{V_{*}}\right),
\end{aligned}
$$

provided $\bar{w}_{\text {reh }} \neq 1 / 3$. As expected, if the reheating is radiation dominated, it cannot be distinguished from the usual radiation era and $\rho_{\text {reh }}$ cannot be inferred. Clearly, for a given set $\left(\epsilon_{1 *}, \epsilon_{2 *}, P_{*}\right)$, in an assumed model of inflation, the right-hand side of Eq. (53) is uniquely determined and hence is $\rho_{\text {reh }}$. As already mentioned, such a method is not well suited: picking up a random $\left(\epsilon_{1 *}, \epsilon_{2 *}, P_{*}\right)$ compatible with the power spectra shapes usually predicts a value of $\rho_{\text {reh }}$ which is either incompatible with $\mathrm{BBN}$, or with the underlying model, i.e. $\rho_{\text {reh }}>\rho_{\text {end }}$. The reason is that inflationary physics is much more than Taylor expanding a potential and fitting the power spectra shape. In order to solve this issue, the way out is to perform an exact numerical integration of the inflationary perturbations, including the reheating parameter, as we previously did. This method has also the advantage to free ourselves from any assumption on the equation of state parameter $\bar{w}_{\text {reh }}$.

\section{CONCLUSION}

We now conclude our investigation by revisiting our main results. The most important conclusion is that, both for large and small field scenarios, the reheating parameter $\ln R$ is now constrained by CMB data. The physical origin of this result is clear. For fixed physical length scales today, a change in $\ln R$ modifies the location of the CMB observable window along the inflationary potential, which is possible only for a limited range of $\ln R$ given the data accuracy. This conclusion is general and does not depend on the details of the reheating epoch. However, if one assumes a model for reheating, typically if one chooses a specific value of the mean equation of state, then it becomes possible to express the constraints mentioned above as limits on the energy density at the end of reheating or, equivalently, as constraints on the reheating temperatures. This leads to Eqs. (46). and (48). These results are of particular interest for the supersymmetric extension of either large or small field inflationary models. Indeed, our result limits the reheating temperature from below whereas gravitinos production gives an upper bound 32 42]: $T_{\text {reh }}<10^{4} \mathrm{TeV}$, where $T_{\text {reh }}$ is given in Eq. (29). Therefore, assuming $g_{*} \simeq 200$ in the large field case, we now have an allowed range of variation for the reheating temperature given by

$$
6 \mathrm{TeV} \lesssim T_{\text {reh }} \lesssim 10^{4} \mathrm{TeV} .
$$


By including the reheating parameter in our analysis, we can marginalize over all reheating history to infer the inflationary parameter values in a robust way. For large field scenarios, we find that the power index is upper limited by $p<2.2$, at $95 \%$ confidence limit. This means that the prototypical model of inflation, namely, massive chaotic inflation, is now under pressure. Similarly, the small field models with sub-Planckian vacuum expectation values $\mu$ are slightly disfavored. In fact, $\mu$ is correlated with the potential power $p$, as represented in Fig. 10. Without marginalization over $p$, large values of $p \gtrsim 6$ are actually necessary to allow the sub-Planckian values of $\mu$ to be inside the $95 \%$ contour. If one has a theoretical prejudice for $\mu<M_{\mathrm{P} l}$, and for reasonable values of $p<6$, then small field models can also be considered under pressure.

Since the constraints on the reheating parameters are directly related to the ability of the data to determine the observable parameters, one could consider more data sets than the WMAP7 data. In fact, since solely the accuracy on the primordial parameters matters, data sets improving the constraints on the standard cosmological parameters do not change the reheating bounds. On the other hand, small scale CMB experiments may be decisive but, as mentioned in Ref. [45], they do not give a significant improvement on the determination of $n_{\mathrm{S}}$ and $r$ due to their low accuracy at large multipoles. We have indeed tested that our limits do not change by including the Baryonic Acoustic Oscillation [60] or the Arcminute Cosmology Bolometer Array Receiver data [61] in our analysis. On the other hand, since the future Planck data are expected to improve the bounds on $n_{\mathrm{S}}, r$ and even on new primordial observables, we should get unprecedented information on the inflationary reheating era.

\section{Acknowledgments}

This work is partially supported by the Belgian Federal Office for Science, Technical and Cultural Affairs, under the Inter-university Attraction Pole Grant No. P6/11.

\section{Appendix A: Reheating from inflaton decay}

For large field models, we have used the fact that $\bar{w}_{\text {reh }}=(p-2) /(p+2)$, a well-known formula established for the first time in Ref. [17]. However, this result assumes that the inflaton field is not coupled to other fields, a hypothesis that, if acceptable at the early stages of the reheating phase, cannot be maintained if one is interested in the transition to the radiation dominated era. The purpose of this Appendix is to take into account the coupling of the inflaton field with the rest of the world and to study its impact on the value of $\bar{w}_{\text {reh }}$. As we show in the following, when the coupling is considered, the value of $\bar{w}_{\text {reh }}$ does not significantly deviate from the equation given above.
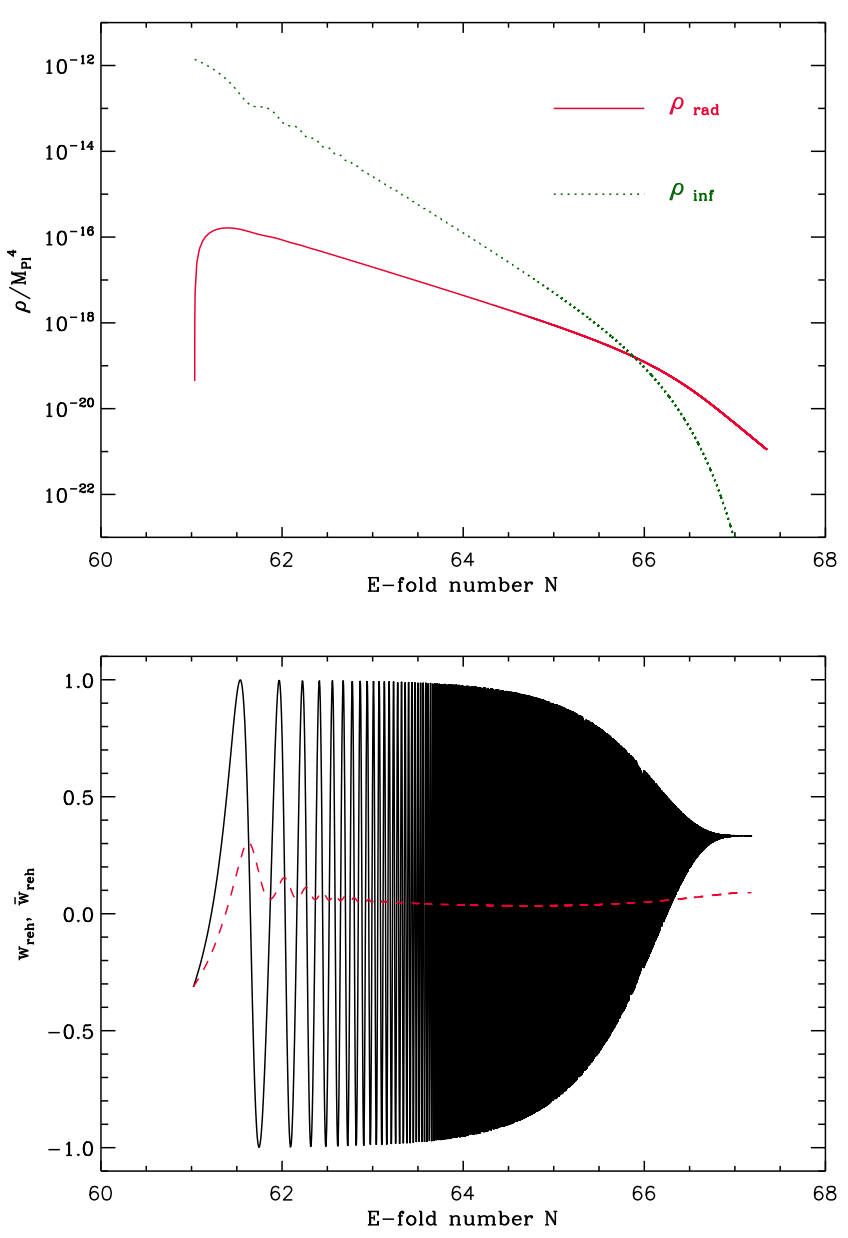

FIG. 13: Top panel: Evolution of the inflaton (dotted green line) and radiation (solid red line) energy densities during the reheating epoch as a function of the number of e-folds since the beginning of large field inflation with $p=2\left(N_{\mathrm{T}} \simeq 61\right)$. The inflaton decay rate has been chosen to be $\Gamma \simeq 1.375 \times$ $10^{9} \mathrm{GeV}$ corresponding to a reheating temperature of $T_{\text {reh }} \simeq$ $3.2 \times 10^{13} g_{*}^{-1 / 4} \mathrm{GeV}$. The total number of e-folds during the reheating epoch is $N_{\text {reh }}=5$. Bottom panel: the instantaneous equation of state (black solid line), $w_{\text {reh }}=\left(P_{\text {inf }}+\rho_{\gamma} / 3\right) /\left(\rho_{\text {inf }}+\right.$ $\left.\rho_{\gamma}\right)$, during the reheating era and going to $1 / 3$. The dashed red line represents $\bar{w}_{\text {reh }}$. Its value when the instantaneous equation of state has reached $1 / 3$ is $\simeq 0.08$ so that, even when the number of e-folds during reheating is small, deviations from $\bar{w}_{\text {reh }}=0$ never exceed $8 \%$.

A simple and standard way to model the physical situation where the inflaton field decays into radiation is to write the Klein-Gordon equation as [17]

$$
\ddot{\phi}+(3 H+\Gamma) \dot{\phi}+\frac{\mathrm{d} V}{\mathrm{~d} \phi}=0
$$

with $\Gamma$ being the inflaton decay rate. The evolution equation for the radiation energy density is modified accordingly in order to ensure that the total energy density is 

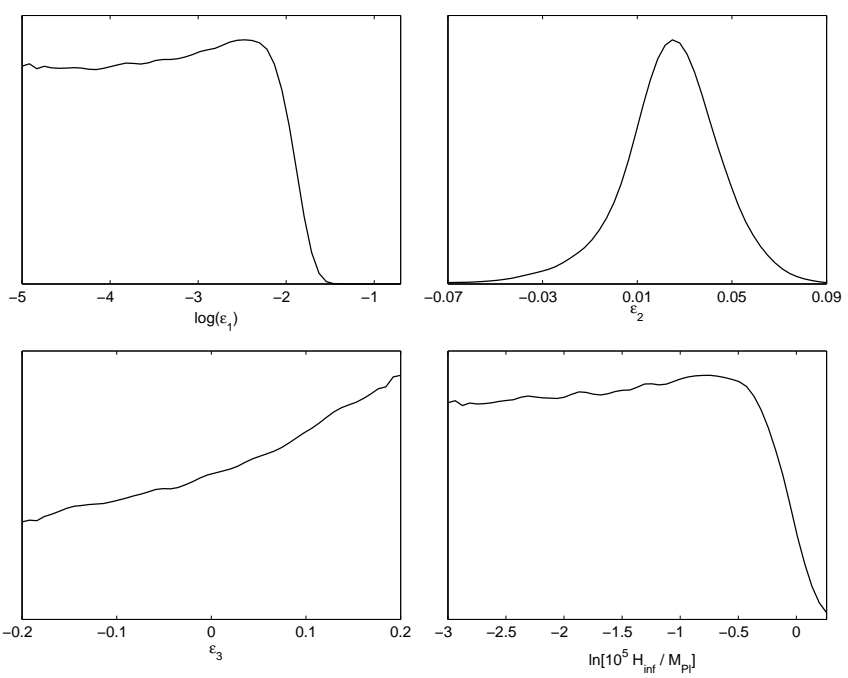

FIG. 14: Marginalized posterior probability distributions (solid lines) and mean likelihoods (dotted lines) for the three first slow-roll parameters and for the energy scale of inflation $\left(\kappa \equiv 1 / M_{\mathrm{P} l}\right)$.

conserved. One obtains

$$
\frac{\mathrm{d} \rho_{\gamma}}{\mathrm{d} N}+4 \rho_{\gamma}=\frac{2 p}{p+2} \frac{\Gamma}{H} \rho_{\mathrm{inf}},
$$

where $\rho_{\text {inf }} \equiv \dot{\phi}^{2} / 2+V(\phi)$. Of course, these two formulas must be supplemented with the Friedmann-Lemaittre equation, $H^{2}=\left(\rho_{\text {inf }}+\rho_{\gamma}\right) /\left(3 M_{\mathrm{pl}}^{2}\right)$. We have numerically integrated Eqs. (A1) and (A2) for large field inflation with $p=2$ and the corresponding evolutions of $\rho_{\text {inf }}$, $\rho_{\text {rad }}, w_{\text {reh }}$ and $\bar{w}_{\text {reh }}$ are displayed in Fig. 13

There are some subtle issues if one wants to numerically evaluate the value of $\bar{w}_{\text {reh }}$. For instance, the time at which one considers the Universe to be reheated is not very well defined since we have a smooth transition, and this affects the precise numerical determination of $\bar{w}_{\text {reh }}$. Indeed, one could consider that reheating is completed when $t \sim \Gamma^{-1}$ or when $w_{\text {reh }}=1 / 3 \pm \delta$ for a given $\delta$. These choices (for instance the precise value of $\delta$ ) lead to different values of the mean equation of state. Moreover, the numerical calculation itself can be difficult since one has to integrate a rapidly oscillating function. All in all, we find that, for $N_{\text {reh }} \simeq 5$ (as in Fig. 13), $\bar{w}_{\text {reh }} \lesssim 0.08$, that is to say a value close to 0 . Let us also notice that when the value of $N_{\text {reh }}$ increases (i.e. when the reheating temperature decreases), one expects this value to be even less than the limit quoted before (but this regime is numerically difficult to follow since the equation of state rapidly oscillates during a long time). Therefore, we conclude that Eq. (23) can reasonably be trusted and this justifies the approach used in this paper.
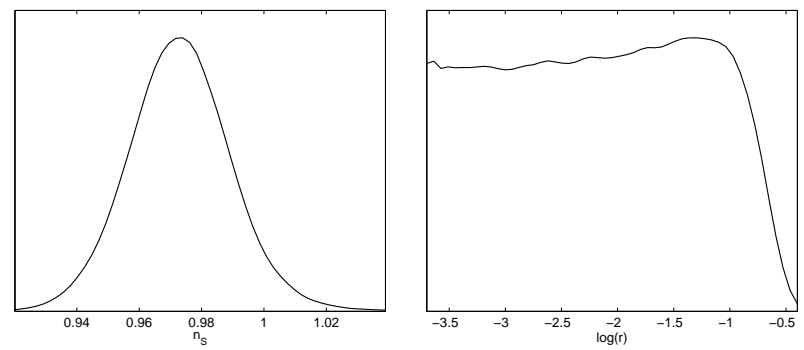

FIG. 15: Marginalized posterior probability distributions (solid lines) for the spectral index and the tensor-to-scalar ratio in the slow-roll approximation. The primordial power spectra are expanded at second order in slow-roll.

\section{Appendix B: Slow-Roll posterior distributions}

In Sec. III we used the one- and two-sigma contours in the planes $\left(n_{\mathrm{S}}, r\right)$ and $\left(\epsilon_{1}, \epsilon_{2}\right)$ obtained from the WMAP7 data. In this section, for the sake of completeness, we also provide the resulting one-dimensional marginalized posterior probability distributions on those four parameters together with the third slow-roll parameters $\epsilon_{3}$ and the energy scale of inflation. Let us notice that our analysis, even for $n_{\mathrm{S}}$ and $r$, assumes the second order slow-roll expanded primordial power spectra for both the scalar and tensor perturbations.

In Fig. 14, we present the marginalized posterior probability distributions for the three first slow-roll parameters and for the Hubble parameter during inflation $\left(H_{\text {inf }}=H_{*}\right)$. For the first slow-roll parameter, we have assumed a Jeffreys' prior in the range $\left[10^{-5}, 0.2\right]$, i.e. a flat prior on $\log \left(\epsilon_{1}\right)$ in the range $[-5,-0.7]$, as appropriate for a parameter the order of magnitude of which is unknown. For the two next slow-roll parameters, we have chosen flat priors in $[-0.2,0.2]$. The upper limit on $\epsilon_{1}$ directly comes from the level of primordial gravitational waves. The parameter $\epsilon_{2}$ is well constrained while $\epsilon_{3}$ remains unbounded. There exists an upper limit on the energy scale of inflation which, as for the first slowroll parameter, directly comes from the non-observation of the primordial gravitational waves. In Fig. 15, the spectral index and the tensor-to-scalar ratio have been plotted and obtained by importance sampling from the slow-roll constraints. Indeed, at second order in slow-roll, one has

$$
\begin{aligned}
n_{\mathrm{S}} & =1-2 \epsilon_{1}-\epsilon_{2}-2 \epsilon_{1}^{2}-(2 C+3) \epsilon_{1} \epsilon_{2}-C \epsilon_{2} \epsilon_{3} \\
r & =16 \epsilon_{1}\left[1+C \epsilon_{2}+\left(C-\frac{\pi^{2}}{2}+5\right) \epsilon_{1} \epsilon_{2}\right. \\
& \left.+\left(\frac{C^{2}}{2}-\frac{\pi^{2}}{8}+1\right) \epsilon_{2}^{2}+\left(\frac{C^{2}}{2}-\frac{\pi^{2}}{24}\right) \epsilon_{2} \epsilon_{3}\right],
\end{aligned}
$$

where $C$ is a numerical constant, $C \simeq-0.7296$. As can be seen on these plots, the spectral index value $n_{\mathrm{S}}=1$ 
is disfavored but not excluded when marginalizing over second order slow-roll. As for $\epsilon_{1}$, there is only an upper limit on the tensor-to-scalar ratio $r$.
[1] A. H. Guth, Phys. Rev. D23, 347 (1981).

[2] A. D. Linde, Phys. Lett. B108, 389 (1982).

[3] A. Albrecht and P. J. Steinhardt, Phys. Rev. Lett. 48, 1220 (1982).

[4] A. D. Linde, Phys. Lett. B129, 177 (1983).

[5] A. D. Linde, Lect. Notes Phys. 738, 1 (2008), 0705.0164.

[6] J. Martin, Braz. J. Phys. 34, 1307 (2004), astro$\mathrm{ph} / 0312492$.

[7] J. Martin, Lect. Notes Phys. 669, 199 (2005), hepth/0406011.

[8] J. Martin, Lect. Notes Phys. 738, 193 (2008), 0704.3540.

[9] V. F. Mukhanov and G. V. Chibisov, JETP Lett. 33, 532 (1981).

[10] S. W. Hawking, Phys. Lett. B115, 295 (1982).

[11] A. A. Starobinsky, Phys. Lett. B117, 175 (1982).

[12] A. H. Guth and S. Y. Pi, Phys. Rev. Lett. 49, 1110 (1982).

[13] J. M. Bardeen, P. J. Steinhardt, and M. S. Turner, Phys. Rev. D28, 679 (1983).

[14] E. D. Stewart and D. H. Lyth, Phys. Lett. B302, 171 (1993), gr-qc/9302019.

[15] V. F. Mukhanov, H. A. Feldman, and R. H. Brandenberger, Phys. Rept. 215, 203 (1992).

[16] A. R. Liddle, P. Parsons, and J. D. Barrow, Phys. Rev. D50, 7222 (1994), astro-ph/9408015.

[17] M. S. Turner, Phys. Rev. D28, 1243 (1983).

[18] L. Kofman, A. D. Linde, and A. A. Starobinsky, Phys. Rev. D56, 3258 (1997), hep-ph/9704452.

[19] B. A. Bassett, S. Tsujikawa, and D. Wands, Rev. Mod. Phys. 78, 537 (2006), astro-ph/0507632.

[20] A. Mazumdar and J. Rocher (2010), 1001.0993.

[21] F. Finelli and R. H. Brandenberger, Phys. Rev. Lett. 82, 1362 (1999), hep-ph/9809490.

[22] B. A. Bassett, D. I. Kaiser, and R. Maartens, Phys. Lett. B455, 84 (1999), hep-ph/9808404.

[23] F. Finelli and R. H. Brandenberger, Phys. Rev. D62, 083502 (2000), hep-ph/0003172.

[24] K. Jedamzik, M. Lemoine, and J. Martin (2010), 1002.3039 .

[25] K. Jedamzik, M. Lemoine, and J. Martin (2010), 1002.3278

[26] R. Easther, R. Flauger, and J. B. Gilmore (2010), 1003.3011.

[27] J. Martin and C. Ringeval, JCAP 0608, 009 (2006), astro-ph/0605367.

[28] K. Nakayama, S. Saito, Y. Suwa, and J. Yokoyama, JCAP 0806, 020 (2008), 0804.1827.

[29] S. Kuroyanagi, C. Gordon, J. Silk, and N. Sugiyama, Phys. Rev. D81, 083524 (2010), 0912.3683.

[30] A. D. Linde, JETP Lett. 38, 176 (1983).

[31] A. D. Linde, Phys. Rev. D49, 748 (1994), astro$\mathrm{ph} / 9307002$.

[32] M. Y. Khlopov and A. D. Linde, Phys. Lett. B138, 265 (1984).

[33] R. Kallosh, L. Kofman, A. D. Linde, and A. Van Proeyen, Phys. Rev. D61, 103503 (2000), hep-th/9907124.
[34] G. F. Giudice, I. Tkachev, and A. Riotto, JHEP 08, 009 (1999), hep-ph/9907510.

[35] M. Lemoine, Phys. Rev. D60, 103522 (1999), hep$\mathrm{ph} / 9908333$.

[36] A. L. Maroto and A. Mazumdar, Phys. Rev. Lett. 84, 1655 (2000), hep-ph/9904206.

[37] G. F. Giudice, A. Riotto, and I. Tkachev, JHEP 11, 036 (1999), hep-ph/9911302.

[38] A. Buonanno, M. Lemoine, and K. A. Olive, Phys. Rev. D62, 083513 (2000), hep-th/0006054.

[39] E. J. Copeland and O. Seto, Phys. Rev. D72, 023506 (2005), hep-ph/0505149.

[40] K. Jedamzik, Phys. Rev. D74, 103509 (2006), hep$\mathrm{ph} / 0604251$.

[41] M. Kawasaki, K. Kohri, T. Moroi, and A. Yotsuyanagi, Phys. Rev. D78, 065011 (2008), 0804.3745.

[42] S. Bailly, K.-Y. Choi, K. Jedamzik, and L. Roszkowski, JHEP 05, 103 (2009), 0903.3974.

[43] J. Mardon, Y. Nomura, and J. Thaler, Phys. Rev. D80, 035013 (2009), 0905.3749.

[44] N. Jarosik et al. (2010), 1001.4744.

[45] E. Komatsu et al. (2010), 1001.4538.

[46] D. Larson et al. (2010), 1001.4635.

[47] C. Ringeval, Lect. Notes Phys. 738, 243 (2008), astro$\mathrm{ph} / 0703486$.

[48] D. J. Schwarz, C. A. Terrero-Escalante, and A. A. Garcia, Phys. Lett. B517, 243 (2001), astro-ph/0106020.

[49] J. Martin and D. J. Schwarz, Phys. Rev. D57, 3302 (1998), gr-qc/9704049.

[50] A. R. Liddle and S. M. Leach, Phys. Rev. D68, 103503 (2003), astro-ph/0305263.

[51] F. Finelli, J. Hamann, S. M. Leach, and J. Lesgourgues (2009), 0912.0522.

[52] C. Ringeval, P. Brax, v. de Bruck, Carsten, and A.-C. Davis, Phys. Rev. D73, 064035 (2006), astro$\mathrm{ph} / 0509727$.

[53] L. Lorenz, J. Martin, and C. Ringeval, JCAP 0804, 001 (2008), 0709.3758.

[54] A. Lewis, A. Challinor, and A. Lasenby, Astrophys. J. 538, 473 (2000), astro-ph/9911177, URL http://camb.info

[55] A. Lewis and S. Bridle, Phys. Rev. D66, $103511 \quad$ (2002), astro-ph/0205436, URL http://cosmologist.info/cosmomc

[56] E. Komatsu and U. Seljak, Mon. Not. Roy. Astron. Soc. 336, 1256 (2002), astro-ph/0205468.

[57] A. G. Riess et al., Astrophys. J. 699, 539 (2009), 0905.0695 .

[58] A. Gelman and D. Rubin, Statistical Science 7, 457 (1992).

[59] L. Lorenz, J. Martin, and C. Ringeval, Phys. Rev. D78, 063543 (2008), 0807.2414.

[60] W. J. Percival et al., Mon. Not. Roy. Astron. Soc. 401, 2148 (2010), 0907.1660.

[61] C. L. Reichardt et al., Astrophys. J. 694, 1200 (2009), 0801.1491 . 